\title{
THE CHEN-RUAN COHOMOLOGY RING OF MIRROR QUINTIC
}

\author{
B. DOUG PARK AND MAINAK PODDAR
}

\begin{abstract}
We compute the Chen-Ruan orbifold cohomology ring of the Batyrev mirror orbifold of a smooth quintic hypersurface in $\mathbb{P}^{4}$. We identify the obstruction bundle for this example by using the Riemann bilinear relations for periods. We outline a general method of computing the Chen-Ruan ring for Calabi-Yau hypersurfaces in projective simplicial toric varieties, modulo a conjecture that the Riemann bilinear relations are adequate for identifying the obstruction bundle for any complex orbifold.
\end{abstract}

\section{INTRODUCTION}

The Chen-Ruan orbifold cohomology (cf. [CR1]) gives mathematical meaning, à la Gromov-Witten theory, to the various invariants such as "orbifold Euler characteristic" and "orbifold cohomology" for global quotient orbifolds that have been in use, courtesy of string theorists, for quite a while. The most interesting feature of this new cohomology theory, besides the generalization to non-global quotients, is the existence of a ring structure which was previously missing. The ring structure is obtained from Chen-Ruan's orbifold quantum cohomology construction (cf. [CR2) by restricting to the class of maps called ghost maps, in the same spirit as the ordinary cup product may be recovered from quantum cup product.

One of the motivations of Chen and Ruan was to construct a mathematical theory that is rich enough to formulate mirror symmetry for Calabi-Yau orbifolds without having to resort to their smooth crepant resolutions which do not always exist in dimensions higher than three. Although the Chen-Ruan cohomology has had several successes, it is yet to make serious progress in this direction. One problem has been that the computation of the ring structure was an unsolved problem when the "obstruction bundle" is not trivial, which is indeed the case for the Batyrev mirror families of hypersurfaces in Fano toric varieties.

Here we try to rectify the situation by suggesting that the Riemann bilinear relations for periods Gr] may be adequate to identify the obstruction bundle. Although we do not prove this, we show that this is true in the mirror quintic example. We then proceed to give an explicit description of the ring structure for this example. The integral of the Euler class of the obstruction bundle is computed first by an ad hoc method that is shorter but rather restrictive, and then recomputed by using localization techniques which should work for any toric Calabi-Yau hypersurface. We deliberately avoid making use of the fact that the mirror quintic is a global quotient. Rather, we focus on developing a strategy that should work in the general setting. For instance the twisted sectors of the mirror quintic can be readily determined since it is a global quotient. However we obtained them as a special

Date: July 4, 2002. Revised on October 10, 2002. 
case of a more general theorem. We would like to point out that the computation of the ordinary cup product of ample toric hypersurfaces is made possible by recent results of Mavlyutov [Ma] and Gröbner basis method (cf. [CLO]).

Finally, we draw the reader's attention to a relevant conjecture of Ruan. String theory suggests that the orbifold quantum cohomology ring of a Calabi-Yau orbifold should be isomorphic to the ordinary quantum cohomology ring of its crepant resolution. This is, of course, not easy to check. It is known that the Chen-Ruan ring structure is not preserved under (partial) crepant resolutions. But the Cohomological Crepant Resolution Conjecture (cf. [Ru]) specifies how the ordinary cup product of the crepant resolution may be deformed by using certain quantum corrections to make it equal to the orbifold cup product of the original space. Developing the machinery to compute the Gromov-Witten invariants involved in these correction terms seems to be an interesting problem. The reader may find a parallel approach to stringy cohomolgy spaces in BM] interesting as well.

Acknowledgments. We thank Alejandro Adem, Karl Heinz Dovermann, Ronald Fintushel, John McCarthy, Yongbin Ruan and Jon Wolfson for very helpful discussions and encouragements. We especially thank Yongbin Ruan for explaining to us how localization might be used to compute integral of the Euler class of the obstruction bundle. The second author also benefitted from conversations with Yuan-Pin Lee, Ernesto Lupercio and Bernardo Uribe. Some computations were done with the aid of Mathematica ${ }^{\circledR}$ Version 4.1 (cf. [Wo]).

\section{Preliminaries}

2.1. Mirror Quintic. Let $\zeta=\exp (2 \pi i / 5)$. Consider the following action of $\left(\mathbb{Z}_{5}\right)^{3}$ on $\mathbb{P}^{4}$ :

$$
\left[x_{1}: x_{2}: x_{3}: x_{4}: x_{5}\right] \mapsto\left[\zeta^{a_{1}} x_{1}: \zeta^{a_{2}} x_{2}: \zeta^{a_{3}} x_{3}: \zeta^{a_{4}} x_{4}: \zeta^{a_{5}} x_{5}\right]
$$

where $a_{i} \in \mathbb{Z}_{5}$ and $\sum_{i=1}^{5} a_{i} \equiv 0 \bmod 5$. From now on, an element of $\left(\mathbb{Z}_{5}\right)^{3}$ will be denoted by a 5 -tuple $g=\left(\zeta^{a_{1}}, \zeta^{a_{2}}, \zeta^{a_{3}}, \zeta^{a_{4}}, \zeta^{a_{5}}\right)$ satisfying the above congruence.

The mirror family (cf. [Bat, , GP]) of smooth quintic hypersurfaces in $\mathbb{P}^{4}$ is given by a one-parameter family of quintic hypersurfaces $X_{\psi} \subset \mathbb{P}^{4} /\left(\mathbb{Z}_{5}\right)^{3}$ satisfying the following equations:

$$
x_{1}^{5}+x_{2}^{5}+x_{3}^{5}+x_{4}^{5}+x_{5}^{5}+\psi x_{1} x_{2} x_{3} x_{4} x_{5}=0 .
$$

Every member of this mirror family is a 3-dimensional Calabi-Yau orbifold, provided that $\psi \neq-5 \zeta^{k}(k \in \mathbb{Z})$. Note that our terminology is slightly different from the existing literature, where the simultaneous desingularization of our family is called the (Batyrev) mirror family. We need to give a toric description of these hypersurfaces. Let $\Delta^{\circ}$ be the polytope in the lattice $N \cong \mathbb{Z}^{4}$ with vertices, $v_{1}=$ $(4,-1,-1,-1), v_{2}=(-1,4,-1,-1), v_{3}=(-1,-1,4,-1), v_{4}=(-1,-1,-1,4)$, $v_{5}=(-1,-1,-1,-1)$. The fan $\Xi$ of the toric variety $\mathbb{P}^{4} /\left(\mathbb{Z}_{5}\right)^{3}$ is obtained by coning over the faces of $\Delta^{\circ}$. Then the $x_{i}$ can be regarded as the generators of the homogeneous coordinate ring of $\mathbb{P}^{4} /\left(\mathbb{Z}_{5}\right)^{3}$ corresponding to the $v_{i}$.

\subsection{Orbifold Structure.}

Definition 2.1. An orbifold structure on a Hausdorff, separable topological space $X$ is given by an open cover $\mathbb{U}$ of $X$ satisfying the following conditions: 
(i) Each element $U$ in $\mathbb{U}$ is uniformized, say by $(V, G, \pi)$. Namely, $V$ is a smooth manifold and $G$ is a finite group acting smoothly on $V$ such that $U=V / G$ with $\pi$ as the quotient map. Let $\operatorname{Ker}(G)$ be the subgroup of $G$ acting trivially on $V$.

(ii) For $U^{\prime} \subset U$, there is a collection of injections $\left(V^{\prime}, G^{\prime}, \pi^{\prime}\right) \rightarrow(V, G, \pi)$. Namely, the inclusion $i: U^{\prime} \subset U$ can be lifted to maps $\tilde{i}: V^{\prime} \rightarrow V$ and an injective homomorphism $i_{*}: G^{\prime} \rightarrow G$ such that $i_{*}$ is an isomorphism from $\operatorname{Ker}\left(G^{\prime}\right)$ to $\operatorname{Ker}(G)$ and $\tilde{i}$ is $i_{*}$-equivariant.

(iii) For any point $x \in U_{1} \cap U_{2}, U_{1}, U_{2} \in \mathbb{U}$, there is a $U_{3} \in \mathbb{U}$ such that $x \in U_{3} \subset U_{1} \cap U_{2}$.

For any point $x \in X$, suppose that $(V, G, \pi)$ is a uniformizing neighborhood and $\bar{x} \in \pi^{-1}(x)$. Let $G_{x}$ be the stabilizer of $G$ at $\bar{x}$. Up to conjugation, it is independent of the choice of $\bar{x}$ and is called the local group of $x$. Then there exists a sufficiently small neighborhood $V_{x}$ of $\bar{x}$ such that $\left(V_{x}, G_{x}, \pi_{x}\right)$ uniformizes a small neighborhood of $x$, where $\pi_{x}$ is the restriction $\left.\pi\right|_{V_{x}}$. $\left(V_{x}, G_{x}, \pi_{x}\right)$ is called a local chart at $x$. The orbifold structure is called reduced if the action of $G_{x}$ is effective for every $x$.

Let $p r: E \rightarrow X$ be a rank $k$ complex orbifold bundle over an orbifold $X$ (cf. [CR1]). Then a uniformizing system for $\left.E\right|_{U}=p r^{-1}(E)$ over a uniformized subset $U$ of $X$ consists of the following data:

(i) A uniformizing system $(V, G, \pi)$ of $U$.

(ii) A uniformizing system $\left(V \times \mathbb{C}^{k}, G, \tilde{\pi}\right)$ for $\left.E\right|_{U}$. The action of $G$ on $V \times \mathbb{C}^{k}$ is an extension of the action of $G$ on $V$ given by $g \cdot(x, v)=(g \cdot x, \rho(x, g) v)$ where $\rho: V \times G \rightarrow \operatorname{Aut}\left(\mathbb{C}^{k}\right)$ is a smooth map satisfying:

$$
\rho(g \cdot x, h) \circ \rho(x, g)=\rho(x, h g), \quad g, h \in G, x \in V .
$$

(iii) The natural projection map $\tilde{p r}: V \times \mathbb{C}^{k} \rightarrow V$ satisfies $\pi \circ \tilde{p r}=p r \circ \tilde{\pi}$.

By an orbifold connection $D$ on $E$ we mean an equivariant connection that satisfies $D=g^{-1} D g$ for every uniformizing system of $E$. Such a connection can be always obtained by the averaging trick and an equivariant partition of unity.

2.3. Twisted Sectors. Let $X$ be an orbifold. Let $\widetilde{X}_{k}$ denote the set of pairs $\left(x,(\mathbf{g})_{x}\right)$ where $(\mathbf{g})_{x}$ stands for the conjugacy class of $\mathbf{g}=\left(g_{1}, \ldots, g_{k}\right)$ with $g_{j} \in G_{x}$. Let $V_{x}^{g_{j}}$ denote the fixed point set of $g_{j}$ in $V_{x}$, and let $C\left(g_{j}\right)$ denote the centralizer of $g_{j}$ in $G_{x} . \widetilde{X}_{k}$ has a natural, possibly nonreduced, orbifold structure (cf. [CR1], [Ka]) whose local chart at $\left(x,(\mathbf{g})_{x}\right)$ is given by

$$
\left(V_{x}^{\mathbf{g}}, C(\mathbf{g}), \pi: V_{x}^{\mathbf{g}} \rightarrow V_{x}^{\mathbf{g}} / C(\mathbf{g})\right)
$$

where $V_{x}^{\mathbf{g}}=\bigcap_{j=1}^{k} V_{x}^{g_{j}}, C(\mathbf{g})=\bigcap_{j=1}^{k} C\left(g_{j}\right)$. The topology on $\widetilde{X}_{k}$ is, of course, specified by declaring each $V_{x}^{\mathbf{g}} / C(\mathrm{~g})$ to be an open set.

We describe the connected components of $\widetilde{X}_{k}$. Each point $x \in X$ has a local chart $\left(V_{x}, G_{x}, \pi_{x}\right)$ which gives a uniformized neighborhood $U_{x}=V_{x} / G_{x}$ of $x$. If $y \in U_{x}$, up to conjugation there is a unique monomorphism $i_{*}: G_{y} \rightarrow G_{x}$. For $\mathrm{g} \in\left(G_{y}\right)^{k}$, the conjugacy class $i_{*}(\mathbf{g})_{y}$ is well-defined. We define an equivalence relation $i_{*}(\mathbf{g})_{y} \cong(\mathbf{g})_{y}$. Let $T_{k}$ denote the set of equivalence classes. By slight abuse of notation, we use $(\mathrm{g})$ to denote the equivalence class to which $(\mathrm{g})_{x}$ belongs. We will usually denote an element of $T_{1}$ by $(g)$. $\tilde{X}_{k}$ is decomposed as a disjoint 
union of connected components

$$
\tilde{X}_{k}=\bigsqcup_{(\mathbf{g}) \in T_{k}} X_{(\mathbf{g})}
$$

where $X_{(\mathbf{g})}=\left\{\left(x,\left(\mathbf{g}^{\prime}\right)_{x}\right) \mid \mathbf{g}^{\prime} \in\left(G_{x}\right)^{k},\left(\mathbf{g}^{\prime}\right)_{x} \in(\mathbf{g})\right\}$. Note that for $\mathbf{g}=(1, \ldots, 1)$ we have $X_{(\mathbf{g})} \cong X$. A component $X_{(\mathbf{g})}$ is called a twisted $k$-sector, provided that $\mathbf{g}$ is not the identity. $X_{(g)}$ is simply called a twisted sector. An almost complex, complex or Kähler structure on $X$ induces an analogous structure on $X_{(\mathrm{g})}$ via (2.2).

Now define

$$
\begin{aligned}
& T_{3}^{0}=\left\{(\mathbf{g})=\left(g_{1}, g_{2}, g_{3}\right) \in T_{3} \mid g_{1} g_{2} g_{3}=1\right\}, \\
& \mathcal{T}_{3}^{0}=\left\{X_{(\mathbf{g})} \mid(\mathbf{g}) \in T_{3}^{0}\right\}
\end{aligned}
$$

There is a one-to-one correspondence between $T_{2}$ and $T_{3}^{0}$ given by $\left(g_{1}, g_{2}\right) \mapsto$ $\left(g_{1}, g_{2},\left(g_{1} g_{2}\right)^{-1}\right)$. We shall call an element of $\mathcal{T}_{3}^{0}$ a tricyclic sector. The twisted sectors of Calabi-Yau hypersurfaces of a Fano toric variety are described in $\mathrm{Po}$. Following the same line of argument, we can identify $\mathcal{T}_{3}^{0}$ in the present example.

\section{Tricyclic Sectors}

3.1. $\mathcal{T}_{3}^{0}$ for Simplicial Toric Varieties. Let $Y$ be the toric variety associated to a simplicial fan $\Xi$ in a $d$-dimensional lattice $N$. Denote the set of $n$-dimensional cones by $\Xi(n)$. For a cone $\tau \in \Xi$, denote the set of its primitive 1-dimensional generators by $\tau[1]$, the corresponding affine open subset of $Y$ by $U_{\tau}$, and the corresponding torus orbit by $O_{\tau}$. We write $\nu \leq \tau$ if the cone $\nu$ is a face of the cone $\tau$, and $\nu<\tau$ if it is a proper subface. $U_{\tau}=\bigsqcup_{\nu \leq \tau} O_{\nu}$. Let $M=\operatorname{Hom}(N, \mathbb{Z})$ be the dual lattice of $N$ with dual pairing $\langle$,$\rangle . For any cone \tau \in \Xi$, denote its dual cone in $M \otimes \mathbb{R}$ by $\check{\tau}$. Let $S_{\tau}=\check{\tau} \cap M$. $\mathbb{C}\left[S_{\tau}\right]$ is the $\mathbb{C}$-algebra with generators $\chi^{m}$ for each $m \in S_{\tau}$ and relations $\chi^{m} \chi^{m^{\prime}}=\chi^{m+m^{\prime}} . U_{\tau}=\operatorname{Spec}\left(\mathbb{C}\left[S_{\tau}\right]\right)$. Define $R(\tau):=\left\{\sum a_{i} f_{i} \mid f_{i} \in \tau[1], 0 \leq a_{i}<1\right\} \cap N$. We will describe the orbifold structure of $Y$.

Let $\sigma$ be any $d$-dimensional cone of $\Xi$. The elements of $\sigma[1], f_{1}, \ldots, f_{d}$, are linearly independent in $N \otimes \mathbb{R}$. Let $N_{\sigma}$ be the sublattice of $N$ generated by $f_{1}, \ldots, f_{d}$. Let $G_{\sigma}:=N / N_{\sigma}$ be the quotient group. $G_{\sigma}$ is finite and Abelian. Let $\sigma^{\prime}$ be the cone $\sigma$ regarded in $N_{\sigma}$. Let $M_{\sigma}$ be the dual lattice of $N_{\sigma}$ and let $\check{\sigma}^{\prime}$ be the dual cone of $\sigma^{\prime}$ in $M_{\sigma} . U_{\sigma^{\prime}}=\operatorname{Spec}\left(\mathbb{C}\left[\check{\sigma}^{\prime} \cap M_{\sigma}\right]\right)$. Note that $\sigma^{\prime}$ is a smooth cone in $N_{\sigma}$. So $U_{\sigma^{\prime}} \cong \mathbb{C}^{d}$.

There is a canonical dual pairing $M_{\sigma} / M \times N / N_{\sigma} \rightarrow \mathbb{Q} / \mathbb{Z} \rightarrow \mathbb{C}^{*}$, the first map by the pairing $\langle$,$\rangle and the second by q \mapsto \exp (2 \pi i q)$. Now $G_{\sigma}$ acts on $\mathbb{C}\left[M_{\sigma}\right]$, the group ring of $M_{\sigma}$, by: $n\left(\chi^{m}\right)=\exp (2 \pi i\langle m, n\rangle) \chi^{m}$, for $n \in N$ and $m \in M_{\sigma}$. Observe that $G_{\sigma}$ acts on $U_{\sigma^{\prime}}$ and $\left(\mathbb{C}\left[M_{\sigma}\right]\right)^{G_{\sigma}}=\mathbb{C}[M]$. Thus $U_{\sigma}=U_{\sigma^{\prime}} / G_{\sigma}$. Let $\pi_{\sigma}$ be the quotient map. So $U_{\sigma}$ is uniformized by $\left(U_{\sigma^{\prime}}, G_{\sigma}, \pi_{\sigma}\right)$. For any $\tau<\sigma$, the orbifold structure on $U_{\tau}$ is same as the one induced from the uniformizing system on $U_{\sigma}$. Then $\left\{\left(U_{\sigma^{\prime}}, G_{\sigma}, \pi_{\sigma}\right) \mid \sigma \in \Xi(d)\right\}$ defines a reduced orbifold structure on $Y$.

It is convenient to have the following description of the local groups. Let $F$ be the nonsingular matrix with generators $f_{1}, \ldots, f_{d}$ of $\sigma$ as rows. Then $\check{\sigma}^{\prime}$ is generated in $M_{\sigma}$ by the column vectors $f^{1}, \ldots, f^{d}$ of the matrix $F^{-1}$. So $\chi^{f^{1}}, \ldots, \chi^{f^{d}}$ are the coordinates of $U_{\sigma^{\prime}}$. For any $r=\left(r_{1}, \ldots, r_{d}\right) \in N$, the corresponding coset $[r] \in G_{\sigma}$ acts on $U_{\sigma^{\prime}}$ in these coordinates as a diagonal matrix: $\operatorname{diag}\left(e^{2 \pi i c_{1}}, \ldots, e^{2 \pi i c_{d}}\right)$ where 
$c_{i}=\left\langle r, f^{i}\right\rangle$. Such a matrix is uniquely represented by a $d$-tuple $a=\left(a_{1}, \ldots, a_{d}\right)$ where $a_{i} \in[0,1)$ and $c_{i}=a_{i}+b_{i}, b_{i} \in \mathbb{Z}$. In matrix notation, $r F^{-1}=a+b \Longleftrightarrow$ $r=a F+b F$. We denote the integral vector $a F$ in $N$ by $r_{a}$ and the diagonal matrix corresponding to $a$ by $g_{a}$. The correspondence $g_{a} \leftrightarrow r_{a}$ gives a bijection between the elements of $G_{\sigma}$ and the elements of $R(\sigma)$.

Now we examine the orbifold chart induced by $\left(U_{\sigma^{\prime}}, G_{\sigma}, \pi_{\sigma}\right)$ at any point $x \in U_{\sigma}$. By the orbit decomposition, there is unique $\tau \leq \sigma$ such that $x \in O_{\tau}$. Assume $\tau$ is generated by $f_{1}, \ldots, f_{j}, j \leq d$. Let $z$ be a preimage of $x$ with respect to $\pi_{\sigma}$. Then $\chi^{f^{i}}(z)=0$ iff $i \leq j$. Let $G_{\tau}:=\left\{g_{a} \in G_{\sigma} \mid a_{i}=0\right.$ if $\left.j+1 \leq i \leq d\right\}=$ $\left\{g_{a} \in G_{\sigma} \mid r_{a} \in R(\tau)\right\}$. We can find a small neighborhood $W \subset\left(\mathbb{C}^{*}\right)^{d-j}$ of $\left(z_{j+1}, \ldots, z_{d}\right)$ such that the inclusions $\mathbb{C}^{j} \times W \hookrightarrow U_{\sigma^{\prime}}$ and $G_{\tau} \hookrightarrow G_{\sigma}$ induces an injection of uniformizing systems $\left(\mathbb{C}^{j} \times W, G_{\tau}, \pi\right) \hookrightarrow\left(U_{\sigma^{\prime}}, G_{\sigma}, \pi_{\sigma}\right)$ on some small open neighborhood $U_{x}$ of $x$. So we have $G_{x}=G_{\tau}$ and an orbifold chart $\left(\mathbb{C}^{j} \times W, G_{\tau}, \pi\right)$.

Now we determine $\mathcal{T}_{3}^{0}$. Take any $x \in Y$ with nontrivial local group. Then $x$ belongs to a unique $O_{\tau}$ such that $\tau$ is not the trivial cone. Pick any elements $g_{a}, g_{b}$ from $G_{x}=G_{\tau}$. We shall find $Y_{(\mathbf{g})}$ where $\mathbf{g}=\left(g_{a}, g_{b},\left(g_{a} g_{b}\right)^{-1}\right)$. Let $\tau_{a}, \tau_{b}$ be the faces of $\tau$, whose interiors contain $r_{a}$ and $r_{b}$ respectively. Let $\sigma$ be any $d$-dimensional cone containing $\tau$. Let $z$ be any point in $U_{\sigma^{\prime}}$. Suppose $z$ is fixed by both $g_{a}$ and $g_{b}$. Then $\chi^{f^{i}}(z)=0$ whenever $f_{i} \in \tau_{a} \cup \tau_{b}$. Hence $\pi_{\sigma}(z) \in \bar{O}_{\tau_{a}} \cap \bar{O}_{\tau_{b}} \cap U_{\sigma}$. A local uniformizing system for $Y_{(\mathbf{g})}$ is given by $\left(V_{x}^{\mathbf{g}}, G_{x}, \pi\right)$, where

$$
V_{x}^{\mathbf{g}}=\left(\mathbb{C}^{j} \times W\right) \cap\left\{\chi^{f^{i}}=0, \forall f_{i} \in \tau_{a} \cup \tau_{b}\right\} .
$$

This leads us to observe that $\left\{(x, \mathbf{g}) \in Y_{(\mathbf{g})} \mid x \in U_{\sigma}\right\}$ is complex analytically isomorphic to $\bar{O}_{\tau_{a}} \cap \bar{O}_{\tau_{b}} \cap U_{\sigma}$. Since this is true irrespective of the choice of $\sigma$, $Y_{\mathbf{g}} \cong \bar{O}_{\tau_{a}} \cap \bar{O}_{\tau_{b}}$. Note that $\bar{O}_{\tau_{1}} \cap \bar{O}_{\tau_{2}}$ is empty whenever $\tau_{1}[1] \cup \tau_{2}[1]$ does not generate an element of $\Xi$.

Proposition 3.1. If $\tau_{1}[1] \cup \tau_{2}[1]$ generate an element of $\Xi$, then for every pair $r_{1} \in R\left(\tau_{1}\right) \cap \operatorname{Int}\left(\tau_{1}\right), r_{2} \in R\left(\tau_{2}\right) \cap \operatorname{Int}\left(\tau_{2}\right)$ we have a unique element of $\mathcal{T}_{3}^{0}(Y)$, which is analytically isomorphic to $\bar{O}_{\tau_{1}} \cap \bar{O}_{\tau_{2}}$. As we vary over $\tau_{1}, \tau_{2}$, we obtain all elements of $\mathcal{T}_{3}^{0}(Y)$.

3.2. $\mathcal{T}_{3}^{0}$ for a Nondegenerate Quasi-smooth Hypersurface. A hypersurface $X$ of the toric variety $Y$ is called quasi-smooth if for any $\sigma \in \Xi(d), \pi_{\sigma}^{-1}\left(X \cap U_{\sigma}\right)$ is smooth. Then $X$ is clearly a suborbifold of $Y$, the orbifold structure being induced by $\left\{\left(\pi_{\sigma}^{-1}\left(X \cap U_{\sigma}\right), G_{\sigma}, \pi_{\sigma}\right) \mid \sigma \in \Xi(d)\right\}$.

$X$ is called nondegenerate if for every $\tau \in \Xi, X \cap O_{\tau}$ is either empty or a smooth variety of codimension one in $O_{\tau}$. When $\mathrm{X}$ is nondegenerate, the above orbifold structure on $X$ is reduced. So any point $x \in X \cap O_{\tau}$ has local group $G_{\tau}$. Then by an argument analogous to the one for $\mathcal{T}_{3}^{0}(Y)$, we have the following description of $\mathcal{T}_{3}^{0}(X)$.

Proposition 3.2. Let $X$ be a nondegenerate quasi-smooth hypersurface of a simplicial toric variety $Y$ with fan $\Xi$. If $\tau_{1}[1] \cup \tau_{2}[1]$ generate an element of $\Xi$, then for every pair $r_{1} \in R\left(\tau_{1}\right) \cap \operatorname{Int}\left(\tau_{1}\right), r_{2} \in R\left(\tau_{2}\right) \cap \operatorname{Int}\left(\tau_{2}\right)$ the connected components of $X \cap \bar{O}_{\tau_{1}} \cap \bar{O}_{\tau_{2}}$ are elements of $\mathcal{T}_{3}^{0}(X)$. As we vary over $\tau_{1}, \tau_{2}$, we obtain all elements of $\mathcal{T}_{3}^{0}(X)$. 
3.3. $\mathcal{T}_{3}^{0}$ for Mirror Quintic. Let $Y=\mathbb{P}^{4} /\left(\mathbb{Z}_{5}\right)^{3}$ and let $X$ be a member of the mirror quintic family such that $\psi \neq-5 \zeta^{k}$ (2.1). Then $X$ is nondegenerate and quasi-smooth. The fan $\Xi$ of $Y$ is described in Subsection 2.1. We shall conveniently use the action of an element of $G_{\tau}$ on the homogeneous coordinate ring of $Y$ to describe that element. For instance, $\left(\zeta, 1, \zeta^{2}, \zeta^{2}, 1\right)$ will represent an element of $G_{\tau}$ where $\tau[1]=\left\{v_{1}, v_{3}, v_{4}\right\}$. The corresponding element of $R_{\tau}$ is $\left(\frac{1}{5} v_{1}+\frac{2}{5} v_{3}+\frac{2}{5} v_{4}\right)$.

Note that $\bar{O}_{\tau}=\left\{x_{i}=0 \mid i\right.$ such that $\left.v_{i} \in \tau[1]\right\}$. Since there is no complex reflection, if $x \in O_{\tau}$ has nontrivial local group then $\operatorname{dim}(\tau) \geq 2$ and consequently $\operatorname{codim}\left(O_{\tau}\right) \geq 2$. By nondegeneracy, $\operatorname{dim}\left(X \cap \bar{O}_{\tau}\right)=\operatorname{codim}(\tau)-1$. Hence a nontrivial element of $\mathcal{T}_{3}^{0}(X)$ (i.e. an element not corresponding to $(\mathbf{g})=(1,1,1)$ ) has dimension less than 2 . We have the following classification of nontrivial elements of $\mathcal{T}_{3}^{0}(X)$.

Corollary 3.3. $X_{(\mathbf{g})}$ is 0 -dimensional if $\tau_{1}[1] \cup \tau_{2}[1]$ generate a 3 -dimensional cone, i.e. if any of the following holds:

(i) $\tau_{1}, \tau_{2}$ are both 2-dimensional and have a 1-dimensional face in common.

(ii) $\tau_{1}$ is a 3-dimensional and $\tau_{2}$ is a 2-dimensional subface of $\tau_{1}$, or vice versa.

(iii) $\tau_{1}=\tau_{2}$ and $\operatorname{dim}\left(\tau_{i}\right)=3$.

$X_{(\mathrm{g})}$ is 1-dimensional if $\tau_{1}[1] \cup \tau_{2}[1]$ generate a 2-dimensional cone, i.e. if $\tau_{1}=\tau_{2}$ and $\operatorname{dim}\left(\tau_{i}\right)=2$.

From the description of $G_{\tau}$ in terms of $R_{\tau}$ in Subsection 3.1, it is not hard to see that $G_{\tau} \cong\left(\mathbb{Z}_{5}\right)^{\operatorname{dim}(\tau)-1}$. For a point $(x,(\mathbf{g})) \in X_{(\mathbf{g})}$ with $x \in O_{\tau} \cap X$ and (g) determined by $r_{i} \in R\left(\tau_{i}\right) \cap \operatorname{Int}\left(\tau_{i}\right)$, a local uniformizing system for $X_{(\mathbf{g})}$ is given by $\left(V_{x}^{\mathbf{g}}, G_{\tau}, \pi\right)$, where $V_{x}^{\mathbf{g}}$ is a small neighborhood of a preimage of $x$ in $\widehat{X} \cap\left\{x_{i}=0, \forall i\right.$ such that $\left.v_{i} \in \tau_{1} \cup \tau_{2}\right\}$. Here $\widehat{X} \subset \mathbb{P}^{4}$ is the zero locus of the polynomial (2.1).

\section{Chen-Ruan Orbifold Cohomology}

Assume that $X$ is a $d$-dimensional compact almost complex orbifold with an almost complex structure $J$ (cf. CR1]). Then for a point $x$ with nontrivial local group $G_{x}, J$ gives rise to an effective representation $\rho_{x}: G_{x} \rightarrow G L(d, \mathbb{C})$. For any $g \in G_{x}$ we write $\rho_{x}(g)$, up to conjugation, as a diagonal matrix

$$
\operatorname{diag}\left(e^{2 \pi i \frac{m_{1, g}}{m_{g}}}, \ldots, e^{2 \pi i \frac{m_{d, g}}{m_{g}}}\right),
$$

where $m_{g}$ is the order of $g$ in $G_{x}$, and $0 \leq m_{i, g}<m_{g}$. Define a function $\iota: \widetilde{X}_{1} \rightarrow \mathbb{Q}$ by

$$
\iota\left(x,(g)_{x}\right)= \begin{cases}\sum_{i=1}^{d} \frac{m_{i, g}}{m_{g}} & \text { if } g \neq 1, \\ 0 & \text { if } g=1 .\end{cases}
$$

This function $\iota: \widetilde{X}_{1} \rightarrow \mathbb{Q}$ is locally constant. Denote its value on $X_{(g)}$ by $\iota_{(g)}$. We call $\iota_{(g)}$ the degree shifting number of $X_{(g)}$. It has the following properties:

(i) $\iota_{(g)}$ is integral iff $\rho_{x}(g) \in S L(d, \mathbb{C})$.

(ii) $\iota_{(g)}+\iota_{\left(g^{-1}\right)}=\operatorname{rank}\left(\rho_{x}(g)-I d\right)=d-\operatorname{dim}_{\mathbb{C}} X_{(g)}$.

A $C^{\infty}$ differential form on $X$ is a $G$-invariant $C^{\infty}$ differential form on $V$ for each uniformizing system $(V, G, \pi)$. Then orbifold integration is defined as follows. 
Suppose $U=V / G$ is connected. For any compactly supported differential $d$-form $\omega$ on $U$, which is, by definition, a $G$-invariant $d$-form $\tilde{\omega}$ on $V$,

$$
\int_{U}^{o r b} \omega:=\frac{1}{|G|} \int_{V} \tilde{\omega}
$$

where $|G|$ is the order of $G$. Then orbifold integration over $X$ is defined by using a $C^{\infty}$ partition of unity. The orbifold integration coincides with the usual measure theoretic integration if and only the orbifold structure on $X$ is reduced.

Holomorphic forms for a complex orbifold $X$ are again obtained by patching $G$ invariant holomorhic forms on the uniformizing systems $(V, G, \pi)$. We consider the Cech cohomology groups of $\mathrm{X}$ and $X_{\mathbf{g}}$ with coeffcients in the sheaves of holomorphic forms. These Cech cohomology groups can be identified with the Dolbeault cohomology groups of $(p, q)$-forms Bai].

Definition 4.1. (cf. CR1) Let $X$ be a closed complex orbifold. We define the orbifold cohomology groups of $X$ by

$$
H_{\text {orb }}^{n}(X):=\bigoplus_{(g) \in T_{1}} H^{n-2 \iota(g)}\left(X_{(g)} ; \mathbb{C}\right) .
$$

We define, for $0 \leq p, q \leq \operatorname{dim}_{\mathbb{C}} X$, orbifold Dolbeault cohomology groups

$$
H_{\text {orb }}^{p, q}(X):=\bigoplus_{(g) \in T_{1}} H^{p-\iota(g), q-\iota(g)}\left(X_{(g)} ; \mathbb{C}\right) .
$$

4.1. Obstruction Bundle. Choose $(\mathbf{g})=\left(g_{1}, g_{2}, g_{3}\right) \in T_{3}^{0}$. Let $\left(x,(\mathbf{g})_{x}\right)$ be a generic point in $X_{(\mathrm{g})}$. Let $K(\mathrm{~g})$ be the subgroup of $G_{x}$ generated by $g_{1}$ and $g_{2}$. Consider an orbifold Riemann sphere with three orbifold points, $\left(\mathbb{S}^{2},\left(x_{1}, x_{2}, x_{3}\right)\right.$, $\left.\left(k_{1}, k_{2}, k_{3}\right)\right)$. When there is no confusion, we will simply denote it by $S^{2}$. The orbifold fundamental group is

$$
\pi_{1}^{\text {orb }}\left(S^{2}\right)=\left\{\lambda_{1}, \lambda_{2}, \lambda_{3} \mid \lambda_{i}^{k_{i}}=1, \lambda_{1} \lambda_{2} \lambda_{3}=1\right\}
$$

where $\lambda_{i}$ is represented by a loop around the marked $x_{i}$. There is a surjective homomorphism

$$
\rho: \pi_{1}^{\text {orb }}\left(S^{2}\right) \rightarrow K(\mathbf{g})
$$

specified by mapping $\lambda_{i} \mapsto g_{i}$. $\operatorname{Ker}(\rho)$ is a finite-index subgroup of $\pi_{1}^{\text {orb }}\left(S^{2}\right)$. Let $\tilde{\Sigma}$ be the orbifold universal cover of $S^{2}$. Let $\Sigma=\tilde{\Sigma} / \operatorname{Ker}(\rho)$. Then $\Sigma$ is smooth, compact and $\Sigma / K(\mathbf{g})=S^{2}$. The genus of $\Sigma$ can be computed using RiemannHurwitz formula for Euler characteristic of a branched cover, and turns out to be

$$
g(\Sigma)=\frac{1}{2}\left(2+|K(\mathbf{g})|-\sum_{i=1}^{3} \frac{|K(\mathbf{g})|}{k_{i}}\right) .
$$

$K(\mathbf{g})$ acts holomorphically on $\Sigma$ and hence $K(\mathbf{g})$ acts on $H^{0,1}(\Sigma)$. The "obstruction bundle" $E_{(\mathbf{g})}$ over $X_{(\mathbf{g})}$ is constructed as follows. On the local chart $\left(V_{x}^{\mathbf{g}}, C(\mathbf{g}), \pi\right)$ of $X_{(\mathbf{g})}, E_{(\mathbf{g})}$ is given by $\left(T V_{x} \otimes H^{0,1}(\Sigma)\right)^{K(\mathbf{g})} \times V_{x}^{\mathbf{g}} \rightarrow V_{x}^{\mathbf{g}}$, where $\left(T V_{x} \otimes H^{0,1}(\Sigma)\right)^{K(\mathbf{g})}$ is the $K(\mathbf{g})$-invariant subspace. We define an action of $C(\mathbf{g})$ on $T V_{x} \otimes H^{0,1}(\Sigma)$, which is the usual one on $T V_{x}$ and trivial on $H^{0,1}(\Sigma)$. Then the actions of $C(\mathbf{g})$ and $K(\mathbf{g})$ commute and $\left(T V_{x} \otimes H^{0,1}(\Sigma)\right)^{K(\mathbf{g})}$ is invariant under $C(\mathbf{g})$. Thus we have obtained an action of $C(\mathbf{g})$ on $\left(T V_{x} \otimes H^{0,1}(\Sigma)\right)^{K(\mathbf{g})} \times V_{x}^{\mathbf{g}} \rightarrow V_{x}^{\mathbf{g}}$, extending 
the usual one on $V_{x}^{\mathbf{g}}$. These trivializations fit together to define the bundle $E_{(\mathrm{g})}$ over $X_{(\mathrm{g})}$. If we set $e: X_{(\mathrm{g})} \rightarrow X$ to be the map given by $\left(x,(\mathrm{~g})_{x}\right) \mapsto x$, one may think of $E_{(\mathbf{g})}$ as $\left(e^{*} T X \otimes H^{0,1}(\Sigma)\right)^{K(\mathbf{g})}$. The rank of $E_{(\mathbf{g})}$ is given by the formula

$$
\operatorname{rank}_{\mathbb{C}}\left(E_{(\mathbf{g})}\right)=\operatorname{dim}_{\mathbb{C}}\left(X_{(\mathbf{g})}\right)-\operatorname{dim}_{\mathbb{C}}(X)+\sum_{j=1}^{3} \iota_{\left(g_{j}\right)} .
$$

4.2. Orbifold Cup Product. There is a natural map $\Phi: X_{(g)} \rightarrow X_{\left(g^{-1}\right)}$ defined by $\left(x,(g)_{x}\right) \mapsto\left(x,\left(g^{-1}\right)_{x}\right)$.

Definition 4.2. Let $d=\operatorname{dim}_{\mathbb{C}}(X)$. For any integer $0 \leq n \leq 2 d$, the pairing

$$
\langle,\rangle_{\text {orb }}: H_{\text {orb }}^{n}(X) \times H_{\text {orb }}^{2 d-n}(X) \longrightarrow \mathbb{C}
$$

is defined by taking the direct sum of

$$
\langle,\rangle_{\text {orb }}^{(g)}: H^{n-2 \iota_{(g)}}\left(X_{(g)} ; \mathbb{C}\right) \times H^{2 d-n-2 \iota_{\left(g^{-1}\right)}}\left(X_{\left(g^{-1}\right)} ; \mathbb{C}\right) \longrightarrow \mathbb{C}
$$

where

$$
\langle\alpha, \beta\rangle_{o r b}^{(g)}=\int_{X_{(g)}}^{o r b} \alpha \wedge \Phi^{*}(\beta)
$$

for $\alpha \in H^{n-2 \iota_{(g)}}\left(X_{(g)} ; \mathbb{C}\right)$, and $\beta \in H^{2 d-n-2 \iota_{\left(g^{-1}\right)}}\left(X_{\left(g^{-1}\right)} ; \mathbb{C}\right)$.

Choose an orbifold connection $A$ on $E_{(\mathbf{g})}$. Let $e_{A}\left(E_{(\mathbf{g})}\right)$ be the Euler form computed from the connection $A$ by Chern-Weil theory. Let $\eta_{j} \in H^{d_{j}}\left(X_{\left(g_{j}\right)} ; \mathbb{C}\right)$, for $j=1,2,3$. Define maps $e_{j}: X_{(\mathbf{g})} \rightarrow X_{\left(g_{j}\right)}$ by $\left(x,(\mathbf{g})_{x}\right) \mapsto\left(x,\left(g_{j}\right)_{x}\right)$.

Definition 4.3. We define the 3-point function to be

$$
\left\langle\eta_{1}, \eta_{2}, \eta_{3}\right\rangle_{\text {orb }}=\int_{X_{(\mathbf{g})}}^{\text {orb }} e_{1}^{*} \eta_{1} \wedge e_{2}^{*} \eta_{2} \wedge e_{3}^{*} \eta_{3} \wedge e_{A}\left(E_{(\mathrm{g})}\right) .
$$

Note that the above integral does not depend on the choice of $A$. As in Definition 4.2, we extend the 3-point function to $H_{o r b}^{*}(X)$ via linearity. We define the orbifold cup product by the relation

$$
\left\langle\eta_{1} \cup_{o r b} \eta_{2}, \eta_{3}\right\rangle_{o r b}=\left\langle\eta_{1}, \eta_{2}, \eta_{3}\right\rangle_{\text {orb }} .
$$

Again we extend $\cup_{o r b}$ to $H_{o r b}^{*}(X)$ via linearity.

Remark 4.4. Note that if $(\mathbf{g})=(1,1,1)$, then $\eta_{1} \cup_{\text {orb }} \eta_{2}$ is just the ordinary cup product $\eta_{1} \cup \eta_{2}$ in $H^{*}(X)$.

\section{Computations for Mirror Quintic}

Let $X$ be a generic member of the mirror quintic family. The crucial part of the cup product computation is identifying the obstruction bundles $E_{(\mathbf{g})}$ and integrating their Euler class. We will content ourselves with computing the 3-point functions. We showed in Subsection 3.2 that $X_{(\mathbf{g})}$ is either a point or a curve, provided that (g) is not the identity. The ordinary cup product corresponding to the identity case will be covered in Section 6 . 
5.1. Point Case. If $X_{(\mathrm{g})}$ is a point then $E_{(\mathrm{g})}$ is a vector space and we can use rank formula (4.4). For the 3-point function to be nonzero, we need $\operatorname{rank}_{\mathbb{C}}\left(E_{(\mathbf{g})}\right)=0$, which forces $\sum_{j=1}^{3} \iota \iota_{\left(g_{j}\right)}=3$. In this case, we have $\left\langle\eta_{1}, \eta_{2}, \eta_{3}\right\rangle_{\text {orb }}=\frac{1}{25} \eta_{1} \eta_{1} \eta_{3}$, where $\eta_{j} \in H^{0,0}\left(X_{\left(g_{j}\right)}\right)$.

All in all there are 930 possible choices of point sectors (g) that give nonzero 3 -point function. We can divide these into two types:

(i) $\iota_{\left(g_{j}\right)}=1$ for all $j=1,2,3$.

(ii) $\iota_{\left(g_{3}\right)}=0$, i.e. $g_{3}=$ id.

Note that for type (i), $\iota_{\left(g_{1} g_{2}\right)}=2$ by property (ii) of degree shifting number in Section 1 . There are 810 point sectors of type (i) and 120 point sectors of type (ii). For example,

$$
\begin{aligned}
& \left(\left(\zeta^{4}, \zeta, 1,1,1\right),\left(1, \zeta^{3}, \zeta^{2}, 1,1\right),\left(\zeta, \zeta, \zeta^{3}, 1,1\right)\right), \\
& \left(\left(\zeta^{3}, \zeta, \zeta, 1,1\right),\left(\zeta, \zeta^{2}, \zeta^{2}, 1,1\right),\left(\zeta, \zeta^{2}, \zeta^{2}, 1,1\right)\right), \\
& \left(\left(\zeta^{2}, \zeta^{3}, 1,1,1\right),\left(\zeta^{2}, \zeta, \zeta^{2}, 1,1\right),\left(\zeta, \zeta, \zeta^{3}, 1,1\right)\right)
\end{aligned}
$$

are type (i) and

$$
\left(\left(\zeta^{3}, \zeta, \zeta, 1,1\right),\left(\zeta^{2}, \zeta^{4}, \zeta^{4}, 1,1\right),(1,1,1,1,1)\right)
$$

is type (ii).

5.2. Curve Case. Now consider the case when $X_{(\mathrm{g})}$ is a curve. Then by Corollary 3.3, up to a canonical isomorphism, $X_{(\mathbf{g})}=\bar{O}_{\tau} \cap X$, where $\operatorname{dim}(\tau)=2$. Consequently $G_{\tau} \cong \mathbb{Z}_{5}$. Hence $K(\mathbf{g})$, the subgroup of $G_{\tau}$ generated by $g_{j}(j=1,2,3)$, is just $G_{\tau}$. Thus we may have $g_{2}=\left(g_{1}\right)^{k}$ where $k=1,2,3,4$. The kernel of the homomorphism $\rho$ (4.2), and hence the Riemann surface $\Sigma$, depend on the value of $k$. We shall fully compute one representative case for each value of $k$. The computations for remaining cases can be completed using the same method and are left to the reader.

In the case $k=4, g_{3}=1$, and so to construct $E_{(\mathrm{g})}$ we have to consider the orbifold sphere $\left(\mathbb{S}^{2},\left(x_{1}, x_{2}, x_{3}\right),(5,5,1)\right)$. Then $\Sigma=\mathbb{S}^{2}$, the smooth sphere.

For other values of $k$, the order of each $g_{j}$ is 5 . So in these cases we need to work with the orbifold sphere $\left(\mathbb{S}^{2},\left(x_{1}, x_{2}, x_{3}\right),(5,5,5)\right)$, which we continue to denote by $S^{2}$. Recall that

$$
\pi_{1}^{\text {orb }}\left(S^{2}\right)=\left\{\lambda_{1}, \lambda_{2}, \lambda_{3} \mid \lambda_{i}^{5}=1, \lambda_{1} \lambda_{2} \lambda_{3}=1\right\} .
$$

Its orbifold universal cover is the hyperbolic plane $\mathbb{H}^{2}$ (cf. $\left.|\mathrm{Sc}|\right)$. We will use the Poincaré disk model of $\mathbb{H}^{2}$. Let $\kappa$ be the geodesic triangle $\triangle$ ovw in $\mathbb{H}^{2}$ as in Figure 1 with all of its angles equal to $\theta=\pi / 5$. The sides of $\kappa$ are labelled by $L, M, N$. Without causing too much confusion, we will denote the hyperbolic reflection about a side of $\kappa$ by the same letter. Let $\epsilon$ denote the region $\kappa \cup M \kappa$.

There is a properly discontinuous action of $\pi_{1}^{o r b}\left(S^{2}\right)$ on $\mathbb{H}^{2}$ which gives a tiling of $\mathbb{H}^{2}$ with fundamental region $\epsilon$ as follows: $\lambda_{1}$ acts as the composition $L \circ M, \lambda_{2}$ acts as the composition $M \circ N$, and $\lambda_{3}$ acts as the composition $N \circ L$. The quotient of $\mathbb{H}^{2}$ by $\pi_{1}^{\text {orb }}\left(S^{2}\right)$ action is the fundamental region $\epsilon$ with the edge $\overline{o u}$ identified with the edge $\overline{o v}$, and the edge $\overline{u w}$ identified with the edge $\overline{v w}$. 


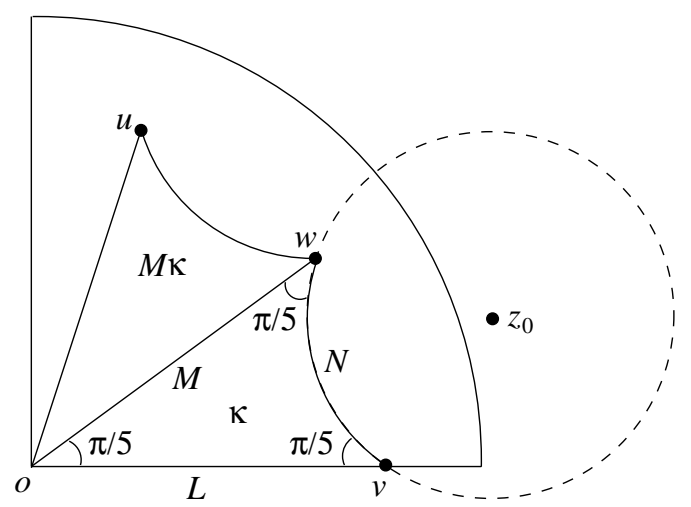

Figure 1. Fundamental region $\epsilon$ in the first quadrant

It is not difficult to compute that

$$
v=\sqrt{\frac{2}{1+\sqrt{5}}} \approx 0.786151 \text {. }
$$

The side $N$ of $\kappa$ is an arc of the circle in $\mathbb{C}$ with center $z_{0}$ and radius $R$, where

$$
\begin{aligned}
& z_{0}=\sqrt{\frac{5+3 \sqrt{5}}{10}} \exp (\pi i / 10) \approx 1.02909+0.33437 i, \\
& R=\operatorname{Im} z_{0} \cdot \sec \theta=\frac{-1+\sqrt{5}}{2 \sqrt[4]{5}} \approx 0.413304 .
\end{aligned}
$$

Then we can write $\lambda_{1}(z)=e^{-i 4 \theta} z$, and

$$
\lambda_{2}(z)=e^{i 4 \theta} \cdot \frac{\overline{z_{0}} z+\left(R^{2}-\left|z_{0}\right|^{2}\right)}{z-z_{0}} .
$$

5.2.1. The Case $g_{1}=g_{2}$. Consider the homomorphism $\rho: \pi_{1}^{\text {orb }}\left(S^{2}\right) \rightarrow K(\mathbf{g})$ given by $\lambda_{i} \mapsto g_{i} \cdot \operatorname{Ker}(\rho)$ is a normal subgroup generated by commutators of $\lambda_{i}$ and the element $\lambda_{1} \lambda_{2}^{-1}$. $\operatorname{Ker}(\rho)$ acts freely on $\mathbb{H}^{2}$ with quotient being the decagon shown in Figure 2. The sides of the decagon are identified by the elements of $\operatorname{Ker}(\rho)$ given in Table 1 .

\section{TABLE 1.}

\begin{tabular}{|c|c|c|c|c|}
\hline$A$ & $B$ & $C$ & $D$ & $E$ \\
\hline$\lambda_{1}^{-1} \lambda_{2}$ & $\lambda_{1} \lambda_{2}^{-1}$ & $\lambda_{1}\left(\lambda_{1} \lambda_{2}^{-1}\right) \lambda_{1}^{-1}$ & $\lambda_{1}^{2}\left(\lambda_{1} \lambda_{2}^{-1}\right) \lambda_{1}^{-2}$ & $\lambda_{1}^{3}\left(\lambda_{1} \lambda_{2}^{-1}\right) \lambda_{1}^{-3}$ \\
\hline
\end{tabular}

We cut out four hyperbolic triangles along the geodesic curves, $\alpha=(B E)^{-1}, \beta=$ $B A, \gamma=B D^{-1}, \delta=E^{-1} C$. After pasting the triangles along the geodesic curves, $A, B, C, D, E$ or their translates by elements of $\operatorname{Ker}(\rho)$, we obtain the octagon in Figure 3, whose boundary curves comprise a symplectic basis $\left\{a_{1}, b_{1}, a_{2}, b_{2}\right\}=$ $\{\alpha, \beta, \gamma, \delta\}$ for $H_{1}(\Sigma ; \mathbb{Z})$ after appropriate identifications. 


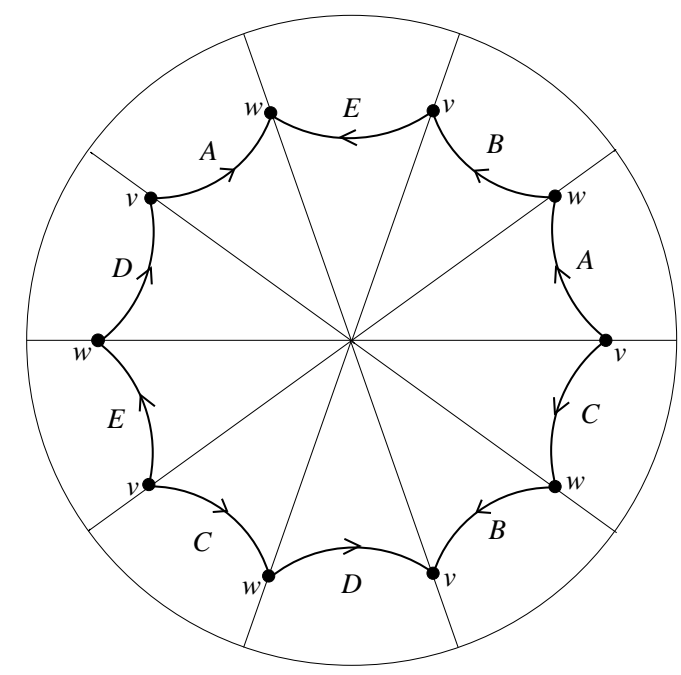

FiguRE 2. Genus two surface $\Sigma$

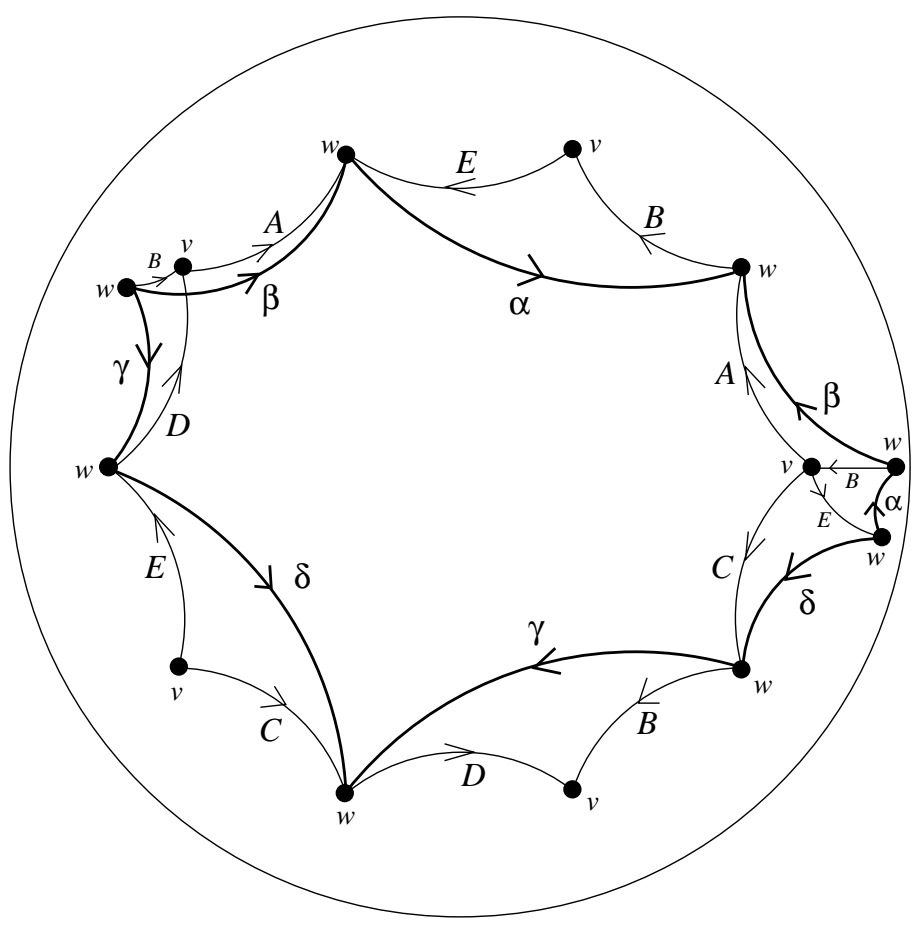

FIGURE 3.

Taking the Universal Coefficients Theorem duals, we obtain a canonical symplectic basis $\{\check{\alpha}, \check{\beta}, \check{\gamma}, \check{\delta}\}$ for $H^{1}(\Sigma ; \mathbb{Z})$. From the Riemann bilinear relations in Gr] (Chapter V, §3), we know that there exists a complex basis $\left\{\omega_{1}, \omega_{2}\right\}$ for $H^{1,0}(\Sigma)=H^{0}\left(\Sigma, K_{\Sigma}\right)$ such that its period matrix with respect to the $a_{i}$-classes 
is the identity matrix, and its period matrix with respect to the $b_{i}$-classes is a symmetric complex matrix

$$
P=\left(\begin{array}{cc}
p & q \\
q & s
\end{array}\right)
$$

with the imaginary part, $\operatorname{Im} P$, being a positive definite matrix. Hence we can write $\left\{\omega_{1}, \omega_{2}\right\}=\{\check{\alpha}+p \check{\beta}+q \check{\delta}, \check{\gamma}+q \check{\beta}+s \check{\delta}\}$.

The rotation $\lambda_{1}$ induces the following map on $\pi_{1}(\Sigma)$ :

$$
\begin{aligned}
& \alpha \longmapsto A^{-1} C=\beta^{-1} \alpha^{-1} \delta \\
& \beta \longmapsto C^{-1} B^{-1}=\delta^{-1} \alpha \\
& \gamma \longmapsto C^{-1} E=\delta^{-1} \\
& \delta \longmapsto A^{-1} D^{-1}=\beta^{-1} \gamma
\end{aligned}
$$

Hence the automorphism $\left(\lambda_{1}\right)_{*}: H_{1}(\Sigma) \rightarrow H_{1}(\Sigma)$ can be expressed in the matrix

$$
\left(\begin{array}{rrrr}
-1 & 1 & 0 & 0 \\
-1 & 0 & 0 & -1 \\
0 & 0 & 0 & 1 \\
1 & -1 & -1 & 0
\end{array}\right)
$$

with respect to the basis $\{\alpha, \beta, \gamma, \delta\}$. The automorphism $\lambda_{1}^{*}: H^{1}(\Sigma) \rightarrow H^{1}(\Sigma)$ can be expressed in the matrix

$$
\left(\begin{array}{rrrr}
-1 & -1 & 0 & 1 \\
1 & 0 & 0 & -1 \\
0 & 0 & 0 & -1 \\
0 & -1 & 1 & 0
\end{array}\right)
$$

with respect to the dual basis $\{\check{\alpha}, \check{\beta}, \check{\gamma}, \check{\delta}\}$, which is the transpose of matrix (5.1). From matrix (5.2), we can easily calculate that

$$
\begin{aligned}
\lambda_{1}^{*} \omega_{1} & =(-1-p+q) \check{\alpha}+(1-q) \check{\beta}+(-q) \check{\gamma}+(-p) \check{\delta}, \\
\lambda_{1}^{*} \omega_{2} & =(-q+s) \check{\alpha}+(-s) \check{\beta}+(-s) \check{\gamma}+(1-q) \check{\delta} .
\end{aligned}
$$

Since $\lambda_{1}$ is a holomorphic map, $\lambda_{1}^{*}$ preserves the subspace $H^{1,0}(\Sigma)$. Hence $\lambda_{1}^{*} \omega_{1}$ and $\lambda_{1}^{*} \omega_{2}$ can be expressed as complex linear combinations of $\omega_{1}$ and $\omega_{2}$, so we easily obtain a system of equations satisfied by the triple $(p, q, s)$ :

$$
\left\{\begin{array}{l}
1-q=p(-1-p+q)-q^{2} \\
-p=q(-1-p+q)-q s \\
-s=p(-q+s)-s q \\
1-q=q(-q+s)-s^{2}
\end{array}\right.
$$

One can verify that there are four solutions to system (5.3), but only one of them satisfies the condition that $\operatorname{Im} P$ is positive definite, namely,

$$
\left\{\begin{array}{l}
p=\exp (3 \pi i / 5), \\
q=\frac{1}{2}(1+i \sqrt{5-2 \sqrt{5}}), \\
s=\exp (2 \pi i / 5)=\zeta
\end{array}\right.
$$


We now know that $H^{0,1}(\Sigma)=\overline{H^{1,0}(\Sigma)}$ is generated by

$$
\left\{\bar{\omega}_{1}, \bar{\omega}_{2}\right\}=\{\check{\alpha}+\bar{p} \check{\beta}+\bar{q} \check{\delta}, \check{\gamma}+\bar{q} \check{\beta}+\bar{s} \check{\delta}\} .
$$

Representative Case. Choose $g_{1}=\left(\zeta^{n}, \zeta^{5-n}, 1,1,1\right)$ where $n=1,2,3$ or 4 . First we consider $e^{*} T X$ over the corresponding $X_{(\mathbf{g})}$, which we identify with $X \cap\left\{x_{1}=\right.$ $\left.x_{2}=0\right\}$ as an analytic space. The orbifold structure on $X_{(\mathbf{g})}$ is not reduced. At the three points $p_{j}=X \cap\left\{x_{1}=x_{2}=x_{j}=0\right\}$ corresponding to $j=3,4$ or 5 , the local groups are isomorphic to $\mathbb{Z}_{5}^{2}$ and contain $K(\mathrm{~g}) \cong \mathbb{Z}_{5}$ as a subgroup. The local group is $K(\mathbf{g})$ at the remaining points. But the $K(\mathbf{g})$ action in the local chart is trivial at each point. By quotienting out all the local groups by $K(\mathbf{g})$, we can associate a reduced orbifold $X_{(\mathbf{g})}^{\prime}:=X_{(\mathbf{g})}^{\text {red }}$ to $X_{(\mathbf{g})}$. We again identify $X_{(\mathbf{g})}^{\prime}$ with $X \cap\left\{x_{1}=x_{2}=0\right\}$, which is a genus zero curve (cf. $|\mathrm{Po}|$, Section 5.3).

Take the open charts $V_{i}=\left\{x_{i} \neq 0\right\}$ of $\mathbb{P}^{4}$, and $U_{i}=V_{i} /\left(\mathbb{Z}_{5}\right)^{3}$ of $\mathbb{P}^{4} /\left(\mathbb{Z}_{5}\right)^{3}$, where $x_{i}$ are the homogeneous coordinates. Let $\pi_{i}: V_{i} \rightarrow U_{i}$ denote the projection map. For points in $V_{5}$, we define new coordinates $z_{i}=x_{i} / x_{5}$, with $z_{5}=1$. On $V_{4}$ we define coordinates $w_{i}=x_{i} / x_{4}$, with $w_{4}=1$. Note that

$$
z_{i}=\frac{x_{i}}{x_{5}}=\frac{x_{i} / x_{4}}{x_{5} / x_{4}}=\frac{w_{i}}{w_{5}} ; \quad w_{i}=\frac{x_{i}}{x_{4}}=\frac{x_{i} / x_{5}}{x_{4} / x_{5}}=\frac{z_{i}}{z_{4}} .
$$

Let $\widehat{X}_{i}=\pi_{i}^{-1}\left(X \cap U_{i}\right)$ and let $X_{(\mathbf{g})}^{\circ}=X_{(\mathbf{g})} \backslash\left\{p_{3}, p_{4}, p_{5}\right\}, X_{(\mathbf{g})}^{\prime \circ}=X_{(\mathbf{g})}^{\prime} \backslash\left\{p_{3}, p_{4}, p_{5}\right\}$.

Next we write down a local framing for $\left.T \widehat{X}_{5}\right|_{\pi_{5}^{-1}\left(X_{(\mathbf{g})}^{\circ}\right)}$ in terms of the $z_{i}$ coordinates. Note that

$$
\widehat{X}_{5}=\left\{z_{1}^{5}+z_{2}^{5}+z_{3}^{5}+z_{4}^{5}+1+\psi z_{1} z_{2} z_{3} z_{4}=0\right\} .
$$

The normal direction to $\widehat{X}_{5}$ on a point is given by the vector

$$
\vec{N}=\left(5 z_{1}^{4}+\psi z_{2} z_{3} z_{4}, 5 z_{2}^{4}+\psi z_{1} z_{3} z_{4}, 5 z_{3}^{4}+\psi z_{1} z_{2} z_{4}, 5 z_{4}^{4}+\psi z_{1} z_{2} z_{3}\right)
$$

For a point $z=\left(0,0, z_{3}, z_{4}\right) \in \pi_{5}^{-1}\left(X_{(\mathbf{g})}^{\circ}\right)$, we have $\vec{N}_{z}=\left(0,0,5 z_{3}^{4}, 5 z_{4}^{4}\right)$. Hence a local framing for $\left.T \widehat{X}_{5}\right|_{\pi_{5}^{-1}\left(X_{(\mathbf{g})}^{\circ}\right)}$ is given by tangent vectors

$$
\left\{\xi_{1}:=\frac{\partial}{\partial z_{1}}, \quad \xi_{2}:=\frac{\partial}{\partial z_{2}}, \quad \xi_{3}:=z_{4}^{4} \frac{\partial}{\partial z_{3}}-z_{3}^{4} \frac{\partial}{\partial z_{4}}\right\} .
$$

$K(\mathbf{g})$ action on the framing is given by $g_{1}\left(z, \xi_{1}\right)=\left(z, \zeta^{n} \xi_{1}\right), g_{1}\left(z, \xi_{2}\right)=\left(z, \zeta^{5-n} \xi_{2}\right)$, and $g_{1}\left(z, \xi_{3}\right)=\left(z, \xi_{3}\right)$.

Now consider the framing $\left\{\xi_{1} \otimes \bar{\omega}_{1}, \xi_{2} \otimes \bar{\omega}_{1}, \xi_{3} \otimes \bar{\omega}_{1}, \xi_{1} \otimes \bar{\omega}_{2}, \xi_{2} \otimes \bar{\omega}_{2}, \xi_{3} \otimes \bar{\omega}_{2}\right\}$ for the bundle $T \widehat{X}_{5} \otimes H^{0,1}(\Sigma)$ over $\pi_{5}^{-1}\left(X_{(\mathbf{g})}^{\circ}\right)$. With respect to this framing, $\lambda_{1}^{*}=g_{1}$ is given by the matrix

$$
\left(\begin{array}{cccccc}
\zeta^{n}(-1-\bar{p}+\bar{q}) & 0 & 0 & \zeta^{n}(-\bar{q}+\bar{s}) & 0 & 0 \\
0 & \zeta^{5-n}(-1-\bar{p}+\bar{q}) & 0 & 0 & \zeta^{5-n}(-\bar{q}+\bar{s}) & 0 \\
0 & 0 & -1-\bar{p}+\bar{q} & 0 & 0 & -\bar{q}+\bar{s} \\
\zeta^{n}(-\bar{q}) & 0 & 0 & \zeta^{n}(-\bar{s}) & 0 & 0 \\
0 & \zeta^{5-n}(-\bar{q}) & 0 & 0 & \zeta^{5-n}(-\bar{s}) & 0 \\
0 & 0 & -\bar{q} & 0 & 0 & -\bar{s}
\end{array}\right)
$$

where $\zeta=\exp (2 \pi i / 5)$. Matrix (5.6) can be diagonalized over $\mathbb{C}$, and we find that 1 is an eigenvalue of multiplicity one with corresponding eigenvector in Table 2 . Hence a generator $s$ for the restricted obstruction bundle $\left.\left(e^{*} T X \otimes H^{0,1}(\Sigma)\right)^{K(\mathbf{g})}\right|_{X_{(\mathbf{g})}^{\circ}} \rightarrow X_{(\mathbf{g})}^{\circ}$ 
TABLE 2 .

\begin{tabular}{|c|c|c|c|}
\hline$n=1$ & $n=2$ & $n=3$ & $n=4$ \\
\hline$\left(0, \zeta^{3}, 0,0,1,0\right)$ & $(0, \zeta, 0,0,1,0)$ & $(\zeta, 0,0,1,0,0)$ & $\left(\zeta^{3}, 0,0,1,0,0\right)$ \\
\hline
\end{tabular}

is given by Table 3. Note however that $s$ does not define an orbifold section of the restricted obstruction bundle.

TABLE 3 .

\begin{tabular}{|l|l|}
\hline$n=1$ & $\zeta^{3}\left(\xi_{2} \otimes \bar{\omega}_{1}\right)+\left(\xi_{2} \otimes \bar{\omega}_{2}\right)$ \\
\hline$n=2$ & $\zeta\left(\xi_{2} \otimes \bar{\omega}_{1}\right)+\left(\xi_{2} \otimes \bar{\omega}_{2}\right)$ \\
\hline$n=3$ & $\zeta\left(\xi_{1} \otimes \bar{\omega}_{1}\right)+\left(\xi_{1} \otimes \bar{\omega}_{2}\right)$ \\
\hline$n=4$ & $\zeta^{3}\left(\xi_{1} \otimes \bar{\omega}_{1}\right)+\left(\xi_{1} \otimes \bar{\omega}_{2}\right)$ \\
\hline
\end{tabular}

Let $\tilde{p}_{3} \in \pi_{5}^{-1}\left(p_{3}\right)$ and $\tilde{p}_{4} \in \pi_{5}^{-1}\left(p_{4}\right)$ be any preimages. Then in a neighborhood of $\tilde{p}_{3}$ or $\tilde{p}_{4},\left(T \widehat{X}_{5} \otimes H^{0,1}(\Sigma)\right)^{K(\mathbf{g})}$ is still generated by the eigenvector from Table 3 . Now consider $p_{5}$ and let $\tilde{p}_{5} \in \pi_{4}^{-1}\left(p_{5}\right)$. Then in a small neighborhood of $\tilde{p}_{5}$ in $\widehat{X}_{4}$, $T \widehat{X}_{4}$ is generated by tangent vectors

$$
\left\{\xi_{1}^{\prime}:=\frac{\partial}{\partial w_{1}}, \quad \xi_{2}^{\prime}:=\frac{\partial}{\partial w_{2}}, \quad \xi_{3}^{\prime}:=w_{5}^{4} \frac{\partial}{\partial w_{3}}-w_{3}^{4} \frac{\partial}{\partial w_{5}}\right\} .
$$

For any point $w=\left(0,0, w_{3}, w_{5}\right)$ in such a neighborhood, $K(\mathbf{g})$ action on this basis is given by $g_{1}\left(w, \xi_{1}^{\prime}\right)=\left(w, \zeta^{n} \xi_{1}^{\prime}\right), g_{1}\left(w, \xi_{2}^{\prime}\right)=\left(w, \zeta^{5-n} \xi_{2}^{\prime}\right)$, and $g_{1}\left(w, \xi_{3}^{\prime}\right)=$ $\left(w, \xi_{3}^{\prime}\right)$. Hence by an argument completely analogous to the $\widehat{X}_{5}$ case, a generator for $\left(T \widehat{X}_{4} \otimes H^{0,1}(\Sigma)\right)^{K(\mathbf{g})}$ can be obtained from Table 3 by substituting $\xi_{j}^{\prime}$ for $\xi_{j}$.

Now we are in a position to describe the local uniformizing charts for $E_{(\mathrm{g})}$. The following description holds only in the cases $n=1$ or 2 . The other two cases are completely analogous and left to the reader.

Choose a local chart $\left(V_{x}^{\mathbf{g}}, C(\mathbf{g}), \pi\right)$ for $X_{(\mathbf{g})}$ at a point $x$.

(i) If $x \in X_{(\mathbf{g})}^{\circ}$, then $C(\mathbf{g})=G_{x}=K(\mathbf{g}) \cong \mathbb{Z}_{5}$, and $\left(V_{x}^{\mathbf{g}} \times \mathbb{C}, K(\mathbf{g}), \tilde{\pi}\right)$ is a uniformizing system for $E_{(\mathbf{g})}$ where $K(\mathbf{g})$ acts on $V_{x}^{\mathbf{g}} \times \mathbb{C}$ by $g_{1}(u, v)=$ $\left(u, \zeta^{5-n} \cdot v\right)$

(ii) If $x=p_{3}$, then $C(\mathbf{g})=G_{x} \cong\left(\mathbb{Z}_{5}\right)^{2}$. An element of $C(\mathbf{g})$ is of the form $\left(\zeta^{a}, \zeta^{b}, \zeta^{c}, 1,1\right)$, where $a+b+c \equiv 0 \bmod 5$. Choose generators $g_{1}$ and $h=\left(1, \zeta, \zeta^{4}, 1,1\right)$ of $C(\mathbf{g}) . C(\mathbf{g})$ acts on $V_{x}^{\mathbf{g}} \times \mathbb{C}$ as follows: $g_{1}(u, v)=$ $\left(u, \zeta^{5-n} \cdot v\right)$, and $h(u, v)=\left(\zeta^{4} \cdot u, \zeta \cdot v\right)$.

(iii) If $x=p_{4}$, then $C(\mathbf{g})=\left\{\left(\zeta^{a}, \zeta^{b}, 1, \zeta^{c}, 1\right) \mid a+b+c \equiv 0 \bmod 5\right\}$. Choose $h=\left(1, \zeta, 1, \zeta^{4}, 1\right)$. Then the $C(\mathbf{g})$ action on $V_{x}^{\mathbf{g}} \times \mathbb{C}$ is given by the same formulas as for $p_{3}$.

(iv) If $x=p_{5}$, then $C(\mathbf{g})=\left\{\left(\zeta^{a}, \zeta^{b}, 1,1, \zeta^{c}\right) \mid a+b+c \equiv 0 \bmod 5\right\}$. Choose $h=\left(1, \zeta, 1,1, \zeta^{4}\right)$. Then the $C(\mathbf{g})$ action on $V_{x}^{\mathbf{g}} \times \mathbb{C}$ is given by the same formulas as for $p_{3}$.

We conclude that there is a smooth $K(\mathbf{g})$ action on $E_{(\mathbf{g})}$ that preserves the fiber. 
$E_{(\mathrm{g})}$ is a line bundle. So the 3-point function (4.5) is nonzero only if $\eta_{i} \in$ $H^{0,0}\left(X_{\left(g_{i}\right)}\right), 1 \leq i \leq 3$. If this is the case then

$$
\left\langle\eta_{1}, \eta_{2}, \eta_{3}\right\rangle_{o r b}=\eta_{1} \eta_{2} \eta_{3} \cdot\left\langle c_{1}\left(E_{(\mathbf{g})}\right),\left[X_{(\mathbf{g})}\right]\right\rangle=\eta_{1} \eta_{2} \eta_{3} \int_{X_{(\mathbf{g})}}^{o r b} c_{1}(A)
$$

where $A$ is any orbifold connection on $E_{(\mathbf{g})}$.

Now consider the associated orbifold principal $S^{1}$ bundle $P_{(\mathrm{g})}$ such that $E_{(\mathrm{g})}=$ $P_{(\mathrm{g})} \times S_{S^{1}} \mathbb{C}$. Recall that there is a global action of $K(\mathrm{~g}) \cong \mathbb{Z}_{5}$ on each fiber $F=S^{1}$ of $P_{(\mathrm{g})}$. The quotient $P_{(\mathrm{g})} / K(\mathrm{~g})$ is again an orbifold principal bundle over the orbifold $X_{(\mathrm{g})}$. Let $\pi_{K(\mathrm{~g})}: P_{(\mathrm{g})} \rightarrow P_{(\mathrm{g})} / K(\mathrm{~g})$ be the quotient map, which extends to an orbifold bundle map. Choose an orbifold connection $A$ that is the pullback $\pi_{K(\mathbf{g})}^{*}\left(A^{\prime}\right)$, where $A^{\prime}$ is an orbifold connection on the associated bundle $E_{(\mathbf{g})}^{\prime}=$ $\left(P_{(\mathrm{g})} / K(\mathbf{g})\right) \times_{S^{1}} \mathbb{C}$ over $X_{(\mathrm{g})}$. Note that $\pi_{K(\mathrm{~g})}$ on each fiber is given by $z \mapsto z^{5}$. The Lie algebra of $F$ can be identified with $\mathbb{R}$. Hence the induced map on the Lie algebra $\left(\pi_{K(\mathbf{g})}\right)_{*}: \mathbb{R} \rightarrow \mathbb{R}$ is just mutiplication by 5 .

Let $\Omega$ and $\Omega^{\prime}$ be curvature 2 -forms for $A$ and $A^{\prime}$. By Proposition 6.1 of [KN], we must have $\pi_{K(\mathbf{g})}^{*}\left(\Omega^{\prime}\right)=5 \cdot \Omega$. Hence by Chern-Weil Theory, we have

$$
\int_{X_{(\mathbf{g})}}^{o r b} c_{1}(A)=\frac{1}{5} \int_{X_{(\mathbf{g})}}^{o r b} c_{1}\left(A^{\prime}\right) \text {. }
$$

Since the action of $K(\mathbf{g})$ in any uniformizing system of $E_{(\mathbf{g})}^{\prime}$ is trivial, $E_{(\mathbf{g})}^{\prime}$ induces an orbifold bundle $E_{(\mathbf{g})}^{\prime \prime}$ over the reduced orbicurve $X_{(\mathbf{g})}^{\prime}$ which has an induced connection $A^{\prime \prime}$. The connections $A^{\prime}$ and $A^{\prime \prime}$ may be represented by the same 1form on $V$ for any pair of corresponding uniformizing systems $\left(V \times \mathbb{C}, G^{\prime}, \tilde{\pi}_{1}^{\prime}\right)$ and $\left(V \times \mathbb{C}, G^{\prime} / K(\mathbf{g}), \tilde{\pi}_{1}^{\prime \prime}\right)$ of $E_{(\mathbf{g})}^{\prime}$ and $E_{(\mathbf{g})}^{\prime \prime}$ respectively. By Chern-Weil theory, $c_{1}\left(A^{\prime}\right)$ and $c_{1}\left(A^{\prime \prime}\right)$ may therefore be represented by the same 2 -form on $V_{1}$. Hence by (4.1)

$$
\int_{X_{(\mathbf{g})}}^{o r b} c_{1}\left(A^{\prime}\right)=\frac{1}{5} \int_{X_{(\mathbf{g})}^{\prime}}^{o r b} c_{1}\left(A^{\prime \prime}\right)=\frac{1}{5}\left\langle c_{1}\left(E_{(\mathbf{g})}^{\prime \prime}\right),\left[X_{(\mathbf{g})}^{\prime}\right]\right\rangle .
$$

Finally we know that (cf. [CR1], equation (4.2.5))

$$
\left.\left\langle c_{1}\left(E_{(\mathbf{g})}^{\prime \prime}\right),\left[X_{(\mathbf{g})}^{\prime}\right]\right\rangle=\left\langle c_{1}\left(\mid E_{(\mathbf{g})}^{\prime \prime}\right)\right),\left[\Sigma_{0}\right]\right\rangle+\sum_{j=3}^{5} \frac{\mu_{j}}{5},
$$

where $\left|E_{(\mathbf{g})}^{\prime \prime}\right|$ denotes the desingularization of $E_{(\mathbf{g})}^{\prime \prime}$ over a smooth genus 0 curve $\Sigma_{0}$, and $0 \leq \mu_{j}<5$ are the local invariants of $E_{(\mathbf{g})}^{\prime \prime}$ at the singular points $p_{j}$ of $X_{(\mathbf{g})}^{\prime}$.

To pin down the local invariants $\mu_{j}$, we need to describe the uniformizing system for $E_{(\mathbf{g})}^{\prime \prime}$. Choose a local chart $\left(V_{x}^{\mathbf{g}}, C(\mathbf{g}) / K(\mathbf{g}), \pi^{\prime \prime}\right)$ for $X_{(\mathbf{g})}^{\prime}$ at a point $x$. In the $n=1$ or 2 cases,

(i) If $x \in X_{(\mathbf{g})}^{\prime}$, then $C(\mathbf{g}) / K(\mathbf{g})$ is the trivial group.

(ii) If $x=p_{3}$, then $C(\mathbf{g}) / K(\mathbf{g}) \cong \mathbb{Z}_{5}$ and $\mathbb{Z}_{5}$ acts on $V_{x}^{\mathbf{g}} \times \mathbb{C}$ by $h(u, v)=$ $\left(\zeta^{4} \cdot u, v\right)$.

From (ii) we immediately conclude that all $\mu_{j}=0$.

To compute $c_{1}\left(\left|E_{(\mathbf{g})}^{\prime \prime}\right|\right)$, note that $s^{5}$ is a holomorphic orbifold section of $\left.E_{(\mathbf{g})}^{\prime \prime}\right|_{X_{(\mathbf{g})}^{\prime}} \rightarrow$ $X_{(\mathrm{g})}^{\prime}$. This section can be extended to a global holomorphic section of the desingularized bundle $\left|E_{(\mathbf{g})}^{\prime \prime}\right|$ as follows. 
Note that we can express our framing (5.5) in terms of $w_{i}$ coordinates. On $V_{4}$ we have

$$
\begin{aligned}
\xi_{1}=\frac{\partial}{\partial z_{1}} & =\sum_{j=1}^{5} \frac{\partial w_{j}}{\partial z_{1}} \cdot \frac{\partial}{\partial w_{j}}=\sum_{j=1}^{5} \frac{\partial}{\partial z_{1}}\left(\frac{z_{j}}{z_{4}}\right) \cdot \frac{\partial}{\partial w_{j}} \\
& =\frac{1}{z_{4}} \cdot \frac{\partial}{\partial w_{1}}=\frac{w_{5}}{w_{4}} \cdot \frac{\partial}{\partial w_{1}}=w_{5} \frac{\partial}{\partial w_{1}} .
\end{aligned}
$$

Similarly, we have

$$
\xi_{2}=\frac{\partial}{\partial z_{2}}=\sum_{j=1}^{5} \frac{\partial w_{j}}{\partial z_{2}} \cdot \frac{\partial}{\partial w_{j}}=\frac{1}{z_{4}} \cdot \frac{\partial}{\partial w_{2}}=w_{5} \frac{\partial}{\partial w_{2}} .
$$

One can express $\xi_{3}$ similarly, but we will not need it.

Now note that $s^{5}$ defines a global holomorphic section of $\left|E_{(\mathbf{g})}^{\prime \prime}\right|$, where

$$
\left.s^{5}\right|_{U_{4} \cap \Sigma_{0}}=\left(\zeta w_{5}\right)^{5} \frac{\partial}{\partial w_{2}} \otimes \bar{\omega}_{1}+\left(w_{5}\right)^{5} \frac{\partial}{\partial w_{2}} \otimes \bar{\omega}_{2} .
$$

We also note that $\left(w_{5}\right)^{5}$ defines the local coordinate of the desingularized curve $\Sigma_{0}$ centered at $p_{5}$. Hence the section $s^{5}$ has a unique zero of order one at the point $p_{5}=\left[x_{1}: x_{2}: x_{3}: x_{4}: x_{5}\right]=[0: 0:-\zeta: 1: 0]$ in $\Sigma_{0}$.

Since a generic holomorphic section of $\left|E_{(\mathbf{g})}^{\prime \prime}\right|$ will also have a single zero, we conclude that

$$
\left\langle c_{1}\left(\left|E_{(\mathbf{g})}^{\prime \prime}\right|\right),\left[\Sigma_{0}\right]\right\rangle=1 .
$$

It follows from (5.8), (5.9) and (5.10) that

$$
\left\langle c_{1}\left(E_{(\mathbf{g})}\right),\left[X_{(\mathbf{g})}\right]\right\rangle=\frac{1}{25} .
$$

Finally, (5.7) becomes

$$
\left\langle\eta_{1}, \eta_{2}, \eta_{3}\right\rangle_{\text {orb }}=\frac{1}{25} \eta_{1} \eta_{2} \eta_{3} .
$$

We remark that (5.13) holds true when $n=3$ or 4 , as well as for the other choices of (g), as long as $g_{2} \neq\left(g_{1}\right)^{4}$.

5.2.2. The Case $g_{2}=\left(g_{1}\right)^{2}$. For this choice of $\rho$, the quotient $\Sigma=\mathbb{H}^{2} / \operatorname{Ker}(\rho)$ is the genus two surface given by the decagon in Figure 1 whose sides are identified according to Table 1 .

\section{TABLE 4.}

\begin{tabular}{|c|c|c|c|c|}
\hline$A$ & $B$ & $C$ & $D$ & $E$ \\
\hline$\lambda_{1}^{-2} \lambda_{2}$ & $\lambda_{2} \lambda_{1}^{-2}$ & $\lambda_{1}^{3}\left(\lambda_{1}^{-2} \lambda_{2}\right) \lambda_{1}^{-3}$ & $\lambda_{1}\left(\lambda_{1}^{-2} \lambda_{2}\right) \lambda_{1}^{-1}$ & $\lambda_{1}^{4}\left(\lambda_{1}^{-2} \lambda_{2}\right) \lambda_{1}^{-4}$ \\
\hline
\end{tabular}

After cutting and pasting along the geodesics as in the previous case, we can find a set of generators of $\pi_{1}(\Sigma)$ based at the point $v$ :

$$
\alpha=A B, \quad \beta=E D C B, \quad \gamma=E D, \quad \delta=C E^{-1}
$$




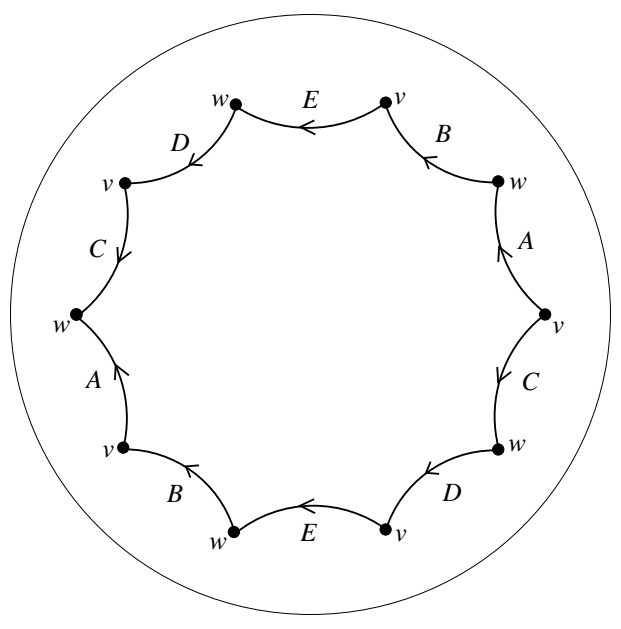

FigURE 4.

which descends to a canonical symplectic basis for $H_{1}(\Sigma ; \mathbb{Z})$. The rotation $\lambda_{1}$ induces the following map on $\pi_{1}(\Sigma)$ :

$$
\begin{aligned}
\alpha & \longmapsto D^{-1} C^{-1}=\gamma^{-1} \delta^{-1} \\
\beta & \longmapsto A B E C^{-1}=\alpha \delta^{-1} \\
\gamma & \longmapsto A B=\alpha \\
\delta & \longmapsto E A^{-1}=\delta^{-1} \gamma^{-1} \beta \alpha^{-1}
\end{aligned}
$$

Hence the automorphism $\lambda_{1}^{*}: H^{1}(\Sigma) \rightarrow H^{1}(\Sigma)$ can be expressed in the matrix

$$
\left(\begin{array}{rrrr}
0 & 1 & 1 & -1 \\
0 & 0 & 0 & 1 \\
-1 & 0 & 0 & -1 \\
-1 & -1 & 0 & -1
\end{array}\right)^{T}=\left(\begin{array}{rrrr}
0 & 0 & -1 & -1 \\
1 & 0 & 0 & -1 \\
1 & 0 & 0 & 0 \\
-1 & 1 & -1 & -1
\end{array}\right)
$$

with respect to the dual basis $\{\check{\alpha}, \check{\beta}, \check{\gamma}, \check{\delta}\}$.

Let $\left\{\omega_{1}, \omega_{2}\right\}=\{\check{\alpha}+p \check{\beta}+q \check{\delta}, \check{\gamma}+q \check{\beta}+s \check{\delta}\}$ be a basis for $H^{1,0}(\Sigma)$ as before. From matrix (5.14), we calculate that

$$
\begin{aligned}
& \lambda_{1}^{*} \omega_{1}=(-q) \check{\alpha}+(1-q) \check{\beta}+\check{\gamma}+(-1+p-q) \check{\delta}=(-q) \omega_{1}+\omega_{2}, \\
& \lambda_{1}^{*} \omega_{2}=(-1-s) \check{\alpha}+(-s) \check{\beta}+(-1+q-s) \check{\delta}=(-1-s) \omega_{1} .
\end{aligned}
$$

Comparing coefficients, we obtain the system:

$$
\left\{\begin{array}{l}
1-q=-q p+q \\
-1+p-q=-q^{2}+s \\
-s=p(-1-s) \\
-1+q-s=q(-1-s)
\end{array}\right.
$$


Just as in the previous case, there are four solutions to system (5.15), but only one of them satisfies the condition that $\operatorname{Im} P$ is positive definite, namely,

$$
\left\{\begin{array}{l}
p=\frac{5-\sqrt{5}}{4}+i \sqrt{\frac{5+\sqrt{5}}{8}}=\sqrt{\frac{5-\sqrt{5}}{2}} \exp (3 \pi i / 10), \\
q=\frac{1}{2}(1+i \sqrt{5-2 \sqrt{5}})=2 \sin (\pi / 10) \cdot \exp (\pi i / 5), \\
s=\frac{-5+\sqrt{5}}{4}+i \sqrt{\frac{5+\sqrt{5}}{8}}=\sqrt{\frac{5-\sqrt{5}}{2}} \exp (7 \pi i / 10) .
\end{array}\right.
$$

Representative Subcase. Without loss of generality, we choose $g_{1}=\left(\zeta^{n}, \zeta^{5-n}, 1,1,1\right)$, where $n=1,2,3,4$. Once again, consider the framing

$$
\left\{\xi_{1} \otimes \bar{\omega}_{1}, \xi_{2} \otimes \bar{\omega}_{1}, \xi_{3} \otimes \bar{\omega}_{1}, \xi_{1} \otimes \bar{\omega}_{2}, \xi_{2} \otimes \bar{\omega}_{2}, \xi_{3} \otimes \bar{\omega}_{2}\right\}
$$

for the bundle $T \widehat{X}_{5} \otimes H^{0,1}(\Sigma)$ over $\pi_{5}^{-1}\left(X_{(\mathbf{g})}^{\circ}\right)$. With respect to this framing, $\lambda_{1}^{*}=g_{1}$ is given by the matrix

$$
\left(\begin{array}{cccccc}
-\zeta^{n} \bar{q} & 0 & 0 & \zeta^{n}(-1-\bar{s}) & 0 & 0 \\
0 & -\zeta^{5-n} \bar{q} & 0 & 0 & \zeta^{5-n}(-1-\bar{s}) & 0 \\
0 & 0 & -\bar{q} & 0 & 0 & -1-\bar{s} \\
\zeta^{n} & 0 & 0 & 0 & 0 & 0 \\
0 & \zeta^{5-n} & 0 & 0 & 0 & 0 \\
0 & 0 & 1 & 0 & 0 & 0
\end{array}\right)
$$

where $\zeta=\exp (2 \pi i / 5)$. Matrix (5.18) is diagonalizable over $\mathbb{C}$, and one finds that 1 is an eigenvalue of multiplicity one with corresponding eigenvector in Table 5 . The

TABLE 5 .

\begin{tabular}{|c|c|c|c|}
\hline$n=1$ & $n=2$ & $n=3$ & $n=4$ \\
\hline$(0, \zeta, 0,0,1,0)$ & $\left(\zeta^{3}, 0,0,1,0,0\right)$ & $\left(0, \zeta^{3}, 0,0,1,0\right)$ & $(\zeta, 0,0,1,0,0)$ \\
\hline
\end{tabular}

rest of the computation goes exactly like in the previous case almost verbatim.

5.2.3. The Case $g_{2}=\left(g_{1}\right)^{3}$. For this choice of $\rho$, the quotient $\Sigma=\mathbb{H}^{2} / \operatorname{Ker}(\rho)$ is the genus two surface given by the decagon in Figure - whose sides are identified according to Table 6 .

TABLE 6 .

\begin{tabular}{|c|c|c|c|c|}
\hline$A$ & $B$ & $C$ & $D$ & $E$ \\
\hline$\lambda_{1}^{-3} \lambda_{2}$ & $\lambda_{2} \lambda_{1}^{-3}$ & $\lambda_{1}^{4}\left(\lambda_{1}^{-3} \lambda_{2}\right) \lambda_{1}^{-4}$ & $\lambda_{1}\left(\lambda_{1}^{-3} \lambda_{2}\right) \lambda_{1}^{-1}$ & $\lambda_{1}^{2}\left(\lambda_{1}^{-3} \lambda_{2}\right) \lambda_{1}^{-2}$ \\
\hline
\end{tabular}

After cutting and pasting along the geodesics as in previous cases, we can find a set of generators of $\pi_{1}(\Sigma)$ based at the point $v$ :

$$
\alpha=E^{-1} C^{-1}, \quad \beta=A B, \quad \gamma=A D, \quad \delta=E^{-1} A^{-1}
$$




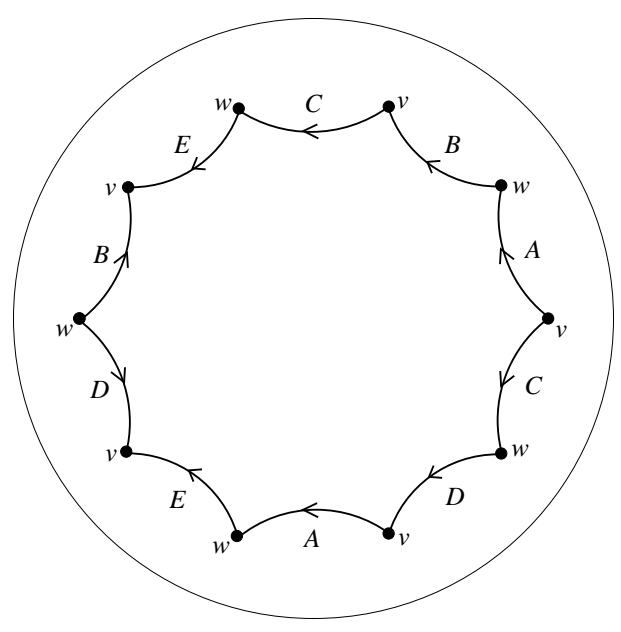

FIGURE 5.

which descends to a canonical symplectic basis for $H_{1}(\Sigma ; \mathbb{Z})$. The rotation $\lambda_{1}$ induces the following map on $\pi_{1}(\Sigma)$ :

$$
\begin{aligned}
& \alpha \longmapsto B^{-1} A^{-1}=\beta^{-1} \\
& \beta \longmapsto D^{-1} C^{-1}=\gamma^{-1} \delta^{-1} \alpha \\
& \gamma \longmapsto D^{-1} E=\gamma^{-1} \delta^{-1} \\
& \delta \longmapsto B^{-1} D=\beta^{-1} \gamma
\end{aligned}
$$

Hence the automorphism $\lambda_{1}^{*}: H^{1}(\Sigma) \rightarrow H^{1}(\Sigma)$ can be expressed in the matrix

$$
\left(\begin{array}{rrrr}
0 & 1 & 0 & 0 \\
-1 & 0 & 0 & -1 \\
0 & -1 & -1 & 1 \\
0 & -1 & -1 & 0
\end{array}\right)^{T}=\left(\begin{array}{rrrr}
0 & -1 & 0 & 0 \\
1 & 0 & -1 & -1 \\
0 & 0 & -1 & -1 \\
0 & -1 & 1 & 0
\end{array}\right)
$$

with respect to the dual basis $\{\check{\alpha}, \check{\beta}, \check{\gamma}, \check{\delta}\}$.

Let $\left\{\omega_{1}, \omega_{2}\right\}=\{\check{\alpha}+p \check{\beta}+q \check{\delta}, \check{\gamma}+q \check{\beta}+s \check{\delta}\}$ be a basis for $H^{1,0}(\Sigma)$ as before. From matrix (5.19), we calculate that

$$
\begin{aligned}
& \lambda_{1}^{*} \omega_{1}=(-p) \check{\alpha}+(1-q) \check{\beta}+(-q) \check{\gamma}+(-p) \check{\delta}=(-p) \omega_{1}+(-q) \omega_{2}, \\
& \lambda_{1}^{*} \omega_{2}=(-q) \check{\alpha}+(-1-s) \check{\beta}+(-1-s) \check{\gamma}+(1-q) \check{\delta}=(-q) \omega_{1}+(-1-s) \omega_{2} .
\end{aligned}
$$

Comparing coefficients, we obtain the system:

$$
\left\{\begin{array}{l}
1-q=-p^{2}-q^{2} \\
-p=-p q-q s \\
-1-s=-p q+q(-1-s) \\
1-q=-q^{2}+s(-1-s)
\end{array}\right.
$$


which has a unique solution satisfying the positive definiteness condition, namely,

$$
\left\{\begin{array}{l}
p=\frac{1}{10} i(5+3 \sqrt{5}) \sqrt{5-2 \sqrt{5}} \\
q=\frac{1}{10}(5-i \sqrt{5(5-2 \sqrt{5})})=\sqrt{\frac{5-\sqrt{5}}{10}} \exp (-\pi i / 10) \\
s=\frac{1}{10}(-5+i \sqrt{5(5+2 \sqrt{5})})=\sqrt{\frac{5+\sqrt{5}}{10}} \exp (7 \pi i / 10) .
\end{array}\right.
$$

Representative Subcase. Without loss of generality, we choose $g_{1}=\left(\zeta^{n}, \zeta^{5-n}, 1,1,1\right)$, where $n=1,2,3,4$. With respect to the framing (5.17) for $T \widehat{X}_{5} \otimes H^{0,1}(\Sigma)$ over $\pi_{5}^{-1}\left(X_{(\mathbf{g})}^{\circ}\right), \lambda_{1}^{*}=g_{1}$ is given by the matrix

$$
\left(\begin{array}{cccccc}
-\zeta^{n} \bar{p} & 0 & 0 & -\zeta^{n} \bar{q} & 0 & 0 \\
0 & -\zeta^{5-n} \bar{p} & 0 & 0 & -\zeta^{5-n} \bar{q} & 0 \\
0 & 0 & -\bar{p} & 0 & 0 & -\bar{q} \\
-\zeta^{n} \bar{q} & 0 & 0 & \zeta^{n}(-1-\bar{s}) & 0 & 0 \\
0 & -\zeta^{5-n} \bar{q} & 0 & 0 & \zeta^{5-n}(-1-\bar{s}) & 0 \\
0 & 0 & -\bar{q} & 0 & 0 & -1-\bar{s}
\end{array}\right)
$$

Matrix (5.22) is diagonalizable, and we find that 1 is an eigenvalue of multiplicity one with corresponding eigenvector in Table 7 .

TABLe 7.

\begin{tabular}{|c|c|}
\hline$n=1$ & $\left(0,2 \operatorname{Re}\left(\zeta^{2}\right), 0,0,1,0\right)$ \\
\hline$n=2$ & $(0,2 \operatorname{Re}(\zeta), 0,0,1,0)$ \\
\hline$n=3$ & $(2 \operatorname{Re}(\zeta), 0,0,1,0,0)$ \\
\hline$n=4$ & $\left(2 \operatorname{Re}\left(\zeta^{2}\right), 0,0,1,0,0\right)$ \\
\hline
\end{tabular}

The rest of the computation goes exactly like in the previous two cases.

5.2.4. The Case $g_{2}=\left(g_{1}\right)^{4}$. As we remarked earlier in this case $\Sigma=\mathbb{S}^{2}$. Hence $H^{0,1}(\Sigma)$ is trivial and $E_{(\mathbf{g})}$ is a rank zero bundle. So $e_{A}\left(E_{(\mathbf{g})}\right)=1$. Thus the 3-point function (4.5)

$$
\left\langle\eta_{1}, \eta_{2}, \eta_{3}\right\rangle_{\text {orb }}=\int_{X_{(\mathbf{g})}}^{\text {orb }} e_{1}^{*} \eta_{1} \wedge e_{2}^{*} \eta_{2} \wedge e_{3}^{*} \eta_{3}
$$

For the integral to be nonzero exactly one of the $\eta_{j}$ must be a $(1,1)$-form and the other two must be 0 -forms.

Let $H$ denote the hyperplane class in $H_{6}\left(\mathbb{P}^{4} / \mathbb{Z}_{5}^{3}\right)$. Let $\eta \in H^{1,1}(X)$ denote the generator whose Poincaré dual is $[H \cap X] \in H_{4}(X)$. Then $\eta^{\prime}=e_{3}^{*} \eta$ generates $H^{1,1}\left(X_{(\mathrm{g})}\right)$. Moreover, the maps $e_{1}$ and $e_{2}$ are isomorphisms in this case. Hence we only need to compute $\int_{X_{(\mathbf{g})}}^{o r b} \eta^{\prime}$.

First write $e_{3}$ as the composition $X_{(\mathbf{g})} \stackrel{q}{\longrightarrow} X_{(\mathbf{g})}^{\prime} \stackrel{j}{\hookrightarrow} X$, where $q$ is the reduction map. We have

$$
\int_{X_{(\mathrm{g})}}^{o r b} e_{3}^{*} \eta=\frac{1}{5} \int_{X_{(\mathbf{g})}^{\prime}}^{o r b} j^{*} \eta=\frac{1}{5} \int_{j\left(X_{(\mathbf{g})}^{\prime}\right)}^{o r b} \eta=\frac{1}{5} \int_{j\left(X_{(\mathbf{g})}^{\prime}\right)} \eta
$$


Note that the last equality follows from the fact that $X_{(\mathbf{g})}^{\prime}$ is reduced.

Since $X$ is a normal complex algebraic variety, there is a well-defined notion of Poincaré duality (cf. [GM]). Hence the last integral is given by the intersection number of $\left[j\left(X_{(\mathbf{g})}^{\prime}\right)\right]$ and $P D(\eta)$; it is easily checked that this is 1 . Hence $\int_{X_{(\mathbf{g})}}^{o r b} \eta^{\prime}=$ $\frac{1}{5}$.

\section{Ordinary Cup Product on Mirror Quintic}

First we determine the Hodge numbers of $X$. We start with Lefschetz Hyperplane Theorem in BC] (Proposition 10.8).

Theorem 6.1. Let $X$ be a nondegenerate ample hypersurface of an n-dimensional complete simplicial toric variety $Y$. Then the natural map induced by inclusion $j^{*}: H^{i}(Y) \rightarrow H^{i}(X)$, is an isomorphism for $i<n-1$ and an injection for $i=n-1$.

Applying the above theorem to our mirror quintic $X$ inside $Y=\mathbb{P}^{4} /\left(\mathbb{Z}_{5}\right)^{3}$, we easily conclude that $h^{1,1}(X)=1$ and $h^{1,0}(X)=h^{2,0}(X)=0$. Complex conjugation gives $h^{0,1}(X)=h^{0,2}(X)=0$. By Kodaira-Serre duality (cf. [CR1], Proposition 3.3.2), it follows that $h^{3,2}(X)=h^{3,1}(X)=0$ and $h^{2,2}(X)=1$. By Theorem 4 in $\left[\mathrm{Po}\right.$, we can calculate that $h^{2,1}(X)=1$.

By Poincaré duality, we may compute the cup product by computing the intersection of dual cycles (cf. GM). Let $H$ denote the hyperplane class in $H_{6}(Y)$. Let $\eta \in H^{1,1}(X)$ denote the generator whose Poincaré dual is $[H \cap X] \in H_{4}(X)$. To compute $\eta \cup \eta \cup \eta$, we look at the intersection of $X$ and three hyperplanes in general position. In particular, we look at hyperplanes $x_{1}=0, x_{2}=0$, and $x_{3}=0$. These intersect $X$ transversally at the unique point $[0: 0: 0:-\zeta: 1]$ in $Y$. Thus $\eta \cup \eta \cup \eta=P D([X])$.

6.1. Middle Cohomology. The cup product for the middle cohomology of an ample hypersurface is described for the smooth case in $\overline{\mathrm{CG}}$ and for the quasismooth case in Ma.

First we state some general facts. Let $X$ be a nondegenerate ample hypersurface in a $d$-dimensional projective simplicial toric variety $Y$. Let $S=\mathbb{C}\left[x_{1}, \ldots, x_{n}\right]$ be the homogeneous coordinate ring of $Y$ which is graded by the Chow group $A_{d-1}(Y)$. Suppose $X$ is defined by a degree $\beta$ homogeneous polynomial $f \in S_{\beta}$. Denote by $D_{i}$ the effective divisor $\left\{x_{i}=0\right\}$ and let $D=\sum b_{i} D_{i}$ be an ample divisor such that $[D]=\beta$. Set $\beta_{0}=\operatorname{deg}\left(x_{1} \ldots x_{n}\right)=\left[\sum_{i=1}^{n} D_{i}\right]$.

Let $F_{j}=x_{j} \frac{\partial f}{\partial x_{j}}$. Given $f \in S_{\beta}$ we get the ideal quotient (cf. [CLO, p. 191)

$$
J_{1}(f)=\left\langle F_{1}, \ldots, F_{n}\right\rangle:\left(x_{1} \ldots x_{n}\right)
$$

and the ring $R_{1}(f)=S / J_{1}(f)$ graded by $A_{d-1}(Y)$.

Fix an integer basis $m_{1}, \ldots, m_{d}$ for the lattice $M$. Moreover suppose $e_{1}, \ldots, e_{n}$ are the primitive integral generators of the 1-dimensional cones of the fan of $Y$. Then given a subset $I=\left\{i_{1}, \ldots, i_{d}\right\} \subset\{1, \ldots, n\}$, we define

$$
\begin{aligned}
\operatorname{det}\left(e_{I}\right) & =\operatorname{det}\left(\left\langle m_{j}, e_{i_{k}}\right\rangle_{1 \leq j \leq d, i_{k} \in I}\right), \\
d x_{I}=d x_{i_{1}} \wedge \ldots \wedge d x_{i_{d}}, \text { and } \hat{x}_{I} & =\prod_{i \notin I} x_{i} . \text { Define the } n \text {-form } \Omega \text { by the formula } \\
\Omega & =\sum_{|I|=d} \operatorname{det}\left(e_{I}\right) \hat{x}_{I} d x_{I} .
\end{aligned}
$$


For $A \in S_{(a+1) \beta-\beta_{0}}$ consider the rational $d$-form $\omega_{A}=A \Omega / f^{a+1}$ which gives a class in $H^{d}(Y-X)$ and by the residue map

$$
\text { Res : } H^{d}(Y-X) \rightarrow H^{d-1}(X)
$$

we get $\operatorname{Res}\left(\omega_{A}\right) \in H^{d-1}(X)$. Denote the $(b, a)$ Hodge component of $\operatorname{Res}\left(\omega_{A}\right)$ by $\operatorname{Res}\left(\omega_{A}\right)^{b, a}$, where $a+b=d-1$. Then the map

$$
\operatorname{Res}\left(\omega_{-}\right)^{b, a}: R_{1}(f)_{(a+1) \beta-\beta_{0}} \rightarrow H^{b, a}(X)
$$

is injective by Theorem 4.4 of [Ma]. By Theorem 11.8 of [BC], the vector spaces $R_{1}(f)_{(a+1) \beta-\beta_{0}}$ and $P H^{b, a}(X):=\left(H^{d-1}(X) / j^{*} H^{d-1}(Y)\right)^{b, a}$ have the same dimension. If $d$ is even then $H^{d-1}(Y)=0$, and hence $P H^{b, a}(X)=H^{b, a}(X)$. Thus for even $d$, which is true for our mirror quintic case, the map (6.1) is an isomorphism.

Now let $I=\left\{i_{0}, \ldots, i_{d}\right\} \subset\{1, \ldots, n\}$. Let $J=\operatorname{det}\left(\frac{\partial F_{j}}{\partial x_{i}}\right)_{i, j \in I} /\left(c_{I}^{\beta}\right)^{2} \hat{x}_{I}$, where $\left(c_{I}^{\beta}\right)$ is the determinant of the $(d+1) \times(d+1)$ matrix obtained from $\left(\left\langle m_{j}, e_{i_{k}}\right\rangle_{1 \leq j \leq d, i_{k} \in I}\right)$ by adding the first row $\left(b_{i_{0}}, \ldots, b_{i_{d}}\right)$.

For $A \in R_{1}(f)_{(a+1) \beta-\beta_{0}}$ and $B \in R_{1}(f)_{(b+1) \beta-\beta_{0}}$ there is a unique constant $c$ such that

$$
A \cdot B\left(x_{1} \ldots x_{n}\right)-c J \in\left\langle x_{1} \frac{\partial f}{\partial x_{1}}, \ldots, x_{n} \frac{\partial f}{\partial x_{n}}\right\rangle .
$$

Then

$$
\int_{X} \operatorname{Res}\left(\omega_{A}\right)^{b, a} \cup \operatorname{Res}\left(\omega_{B}\right)^{a, b}=c(-2 \pi i)^{d} c_{a b} d ! \operatorname{Vol}\left(\Delta_{D}\right),
$$

where $c_{a b}=\frac{(-1)^{a(a+1) / 2+b(b+1) / 2+a^{2}+d-1}}{a ! b !}$ and $\Delta_{D}$ is the polytope associated to the ample divisor $D$.

In the mirror quintic situation, $n=d+1=5, e_{i}=v_{i}, \beta=\beta_{0}=\left[\sum_{i=1}^{5} D_{i}\right]$. $\Delta_{D}$ is the polytope with vertices $(1,0,0,0),(0,1,0,0),(0,0,1,0),(0,0,0,1)$ and $(-1,-1,-1,-1)$. Note that $\operatorname{Vol}\left(\Delta_{D}\right)=\frac{5}{4 !}$. We compute that $\left(c_{I}^{\beta}\right)=625$, and

$$
\begin{aligned}
J= & \psi\left(x_{1} x_{2} x_{3} x_{4}\right)^{5}+\psi\left(x_{1} x_{2} x_{3} x_{5}\right)^{5}+\psi\left(x_{1} x_{2} x_{4} x_{5}\right)^{5} \\
& +\psi\left(x_{1} x_{3} x_{4} x_{5}\right)^{5}+\psi\left(x_{2} x_{3} x_{4} x_{5}\right)^{5}+25\left(x_{1} x_{2} x_{3} x_{4} x_{5}\right)^{4} .
\end{aligned}
$$

Now there are two cases to consider.

(i) $H^{3,0} \cup H^{0,3}$ : Both $H^{3,0}(X) \cong R_{1}(f)_{0}$ and $H^{1,2}(X) \cong R_{1}(f)_{3 \beta}$ have rank 1 . Choose $A=1 \in R_{1}(f)_{0}$ and $B^{\prime}=\left(x_{1} x_{2} x_{3} x_{4} x_{5}\right)^{3} \in R_{1}(f)_{3 \beta}$. Then we use the Division Algorithm in CLO, p. 61, to compute that $c=\frac{125}{\psi^{5}+5^{5}}$. Here we have $a=0, b=3$ and thus $c_{a b}=-\frac{1}{3 !}$. Hence we have

$$
\operatorname{Res}\left(\omega_{A}\right)^{3,0} \cup \operatorname{Res}\left(\omega_{B}\right)^{0,3}=-\frac{5000 \pi^{4}}{3\left(\psi^{5}+5^{5}\right)} P D([X]) .
$$

(ii) $H^{2,1} \cup H^{1,2}$ : Again both $H^{2,1}(X) \cong R_{1}(f)_{\beta}$ and $H^{1,2}(X) \cong R_{1}(f)_{2 \beta}$ have rank 1. Choose $A^{\prime}=x_{1} x_{2} x_{3} x_{4} x_{5} \in R_{1}(f)_{\beta}$ and $B^{\prime}=\left(x_{1} x_{2} x_{3} x_{4} x_{5}\right)^{2} \in$ $R_{1}(f)_{2 \beta}$. Since $A^{\prime} \cdot B^{\prime}$ is same as $A \cdot B$, we have the same $c=\frac{125}{\psi^{5}+5^{5}}$. But now $a=1, b=2$ and thus $c_{a b}=\frac{1}{2 !}$. Hence we have

$$
\operatorname{Res}\left(\omega_{A^{\prime}}\right)^{2,1} \cup \operatorname{Res}\left(\omega_{B^{\prime}}\right)^{1,2}=\frac{5000 \pi^{4}}{\psi^{5}+5^{5}} P D([X]) .
$$




\section{3-Point Function via Localization}

Consider the general case, i.e. $X$ is a nondegenerate Calabi-Yau hypersurface of a projective simplicial toric variety $Y$. Then a tricyclic sector $X_{(\mathrm{g})}$ is a hypersurface of the corresponding tricyclic sector $Y_{(\mathbf{g})}$ of $Y$. Denote the associated reduced varieties by $X_{(\mathbf{g})}^{\prime}$ and $Y_{(\mathbf{g})}^{\prime}$. We showed that $Y_{(\mathbf{g})}^{\prime}$ is a toric variety. There exists a torus equivariant line bundle $L_{(\mathrm{g})}$ over $Y_{(\mathbf{g})}^{\prime}$ with $X_{(\mathrm{g})}^{\prime}$ as the zero locus of a section of $L_{(\mathrm{g})}$. On the other hand, the obstruction bundle $F_{(\mathrm{g})}$ over $Y_{(\mathrm{g})}$ restricts to the reduced obstruction bundle $E_{(\mathrm{g})}$ over $X_{(\mathrm{g})}$. This follows from the definition of obstruction bundle and the rank formula (4.4). We can associate reduced bundles $E_{(\mathrm{g})}^{\prime \prime}$ over $X_{(\mathbf{g})}^{\prime}$ to $E_{(\mathbf{g})}$ and $F_{(\mathbf{g})}^{\prime \prime}$ over $Y_{(\mathbf{g})}^{\prime}$ to $F_{(\mathbf{g})}$ by using a $K(\mathbf{g})$-invariant homomorphism of the structure group as in Section $5 . F_{(\mathbf{g})}^{\prime \prime}$ restricts to $E_{(\mathbf{g})}^{\prime \prime}$ over $X_{(\mathbf{g})}^{\prime}$. By Poincaré duality we have

$$
\int_{X_{(\mathbf{g})}^{\prime}} e\left(E_{(\mathbf{g})}^{\prime \prime}\right)=\int_{Y_{(\mathbf{g})}^{\prime}} e\left(F_{(\mathbf{g})}^{\prime \prime}\right) \wedge e\left(L_{(\mathbf{g})}\right)=\int_{Y_{(\mathbf{g})}^{\prime}} e\left(F_{(\mathbf{g})}^{\prime \prime} \oplus L_{(\mathbf{g})}\right)
$$

The torus action on $Y_{(\mathbf{g})}^{\prime}$ lifts to an action (not unique) on the bundle $F_{(\mathbf{g})}^{\prime \prime} \oplus L_{(\mathbf{g})}$ that maps each fiber linearly. Hence we can use the localization technique of Atiyah and Bott AB, adapted to reduced orbifolds, to calculate the last integral in (7.1). We carry this out below when $X \subset Y=\mathbb{P}^{4} /\left(\mathbb{Z}_{5}\right)^{3}$ is a member of the mirror quintic family. Let $\Xi$ denote the fan of $\mathbb{P}^{4} /\left(\mathbb{Z}_{5}\right)^{3}$ in $N \otimes \mathbb{R}$ as in Section 2.1 and let $M$ denote the dual lattice of $N$.

Let $g_{1}=\left(\zeta^{2}, \zeta^{3}, 1,1,1\right)$ and $g_{2}=g_{1}$. Then $Y_{(\mathrm{g})}^{\prime}$ is isomorphic to the toric variety $\left\{x_{1}=x_{2}=0\right\} \cap Y$. In other words, $Y_{(\mathbf{g})}^{\prime}=\bar{O}_{\tau}$ where $\tau[1]=\left\{v_{1}, v_{2}\right\}$. Recall that $N_{\tau}$ is the sublattice of $N$ generated by $v_{1}$ and $v_{2}$. Let $N(\tau)=N / N_{\tau}$ be the quotient lattice. The fan of $Y_{(\mathbf{g})}^{\prime}$ is given by the projection of $\Xi$ to $N(\tau) \otimes \mathbb{R}$. The dual lattice of $N(\tau)$ is $M(\tau)=\tau^{\perp} \cap M$. The 2-dimensional torus associated to $Y_{(\mathbf{g})}^{\prime}$ is $\mathbf{T}=\operatorname{Spec}(\mathbb{C}[M(\tau)])=O_{\tau}$. The characters $\chi^{m}$ correspond to rational functions on $Y_{(\mathbf{g})}^{\prime}$ when $m \in M(\tau)$. Let $\left\{m_{1}, m_{2}, m_{3}, m_{4}\right\}$ be the standard basis of $M$. Then $\left\{c_{1}=m_{1}+m_{2}+3 m_{4}, c_{2}=m_{3}-m_{4}\right\}$ is a basis for $M(\tau)$. The $\mathbf{T}$ action on $Y_{(\mathbf{g})}^{\prime}$ has three fixed points $q_{j}=\left\{x_{i}=\delta_{i, j}\right\}, 3 \leq j \leq 5$. We study the action of $\mathbf{T}$ on the normal bundle of $q_{j}$, i.e., the orbifold tangent space $\left(T Y_{(\mathrm{g})}^{\prime}\right)_{q_{j}}$.

Consider $q_{5}$ first. Denote local coordinates on a uniformizing sysytem of $Y_{(\mathrm{g})}^{\prime}$ around $q_{5}$ by $z_{3}=\frac{x_{3}}{x_{5}}$ and $z_{4}=\frac{x_{4}}{x_{5}}$ as in Section 5. Let $m^{1}=\frac{1}{5} c_{1}+\frac{2}{5} c_{2}$ and $m^{2}=\frac{1}{5} c_{1}+\frac{1}{5} c_{2}$. Then observe that $\left\langle m^{1}, v_{3}\right\rangle=1,\left\langle m^{1}, v_{4}\right\rangle=0$ and $\left\langle m^{1}, v_{5}\right\rangle=-1$. Hence $z_{3}=\chi^{m^{1}}$ (see Section 3.1). Similarly, $z_{4}=\chi^{m^{2}}$. Thus with respect to the basis $\left\{c_{1}, c_{2}\right\}, \mathbf{T}$ action is given by $\left(t_{1}, t_{2}\right)\left(z_{3}\right)=t_{1}^{\frac{1}{5}} t_{2}^{\frac{2}{5}} z_{3}$ and $\left(t_{1}, t_{2}\right)\left(z_{4}\right)=t_{1}^{\frac{1}{5}} t_{2}^{\frac{1}{5}} z_{4}$. Let $u_{1}, u_{2}$ be parameters on the Lie algebra $\mathbf{t}_{\mathbb{C}}$ of $\mathbf{T}$ corresponding the above choice of basis. Then the T-equivariant Euler class of the normal bundle of $q_{5}$ is given by $e_{\mathbf{T}}\left(\nu_{q_{5}}\right)=\left(\frac{1}{5} u_{1}+\frac{2}{5} u_{2}\right)\left(\frac{1}{5} u_{1}+\frac{1}{5} u_{2}\right)$. One similarly obtains $e_{\mathbf{T}}\left(\nu_{q_{4}}\right)=$ $\left(\frac{1}{5} u_{2}\right)\left(-\frac{1}{5} u_{1}-\frac{1}{5} u_{2}\right)$, and $e_{\mathbf{T}}\left(\nu_{q_{3}}\right)=\left(-\frac{1}{5} u_{2}\right)\left(-\frac{1}{5} u_{1}-\frac{2}{5} u_{2}\right)$.

Now we want to lift the $\mathbf{T}$ action to the line bundles $L_{(\mathbf{g})}$ and $F_{(\mathbf{g})}^{\prime \prime}$. Quite generally, suppose $L$ is a line bundle on a toric variety, corresponding to a Cartier divisor $\left\{U_{\sigma}, \chi^{-m_{\sigma}}\right\}$. The transition functions $h_{\tau \sigma}: U_{\sigma} \times \mathbb{C} \supset U_{\sigma \cap \tau} \times \mathbb{C} \rightarrow U_{\sigma \cap \tau} \times$ $\mathbb{C} \subset U_{\tau} \times \mathbb{C}$ for $L$ are given by $h_{\tau \sigma}(x, c)=\left(x, \chi^{\left(m_{\sigma}-m_{\tau}\right)}(x) c\right)$. Then one can 
define a $\mathbf{T}$ action on $L$ that makes it a $\mathbf{T}$-equivariant bundle (cf. $\mathrm{Od}$ ) as follows: $t(x, c)=\left(t x, \chi^{-m_{\sigma}}(t) c\right)$ for $t \in \mathbf{T}$ and $(x, c) \in U_{\sigma} \times \mathbb{C}$.

Let $D_{i}$ denote the Weil divisor $\left\{x_{i}=0\right\}$ of $Y$. Then the hypersurface $X$ corresponds to a section of the anticanonical bundle $-K_{Y}=\sum_{i=1}^{5} D_{i}$. Thus $X \cap\left\{x_{1}=x_{2}=0\right\}$ corresponds to a section of $-K_{Y} D_{1} D_{2}$ which is linearly equivalent to $5 D_{1} D_{2} D_{3}$. Hence $L_{(\mathrm{g})}=\left[5 D_{1} D_{2} D_{3}\right]$. Consider the open covering of $Y_{(\mathrm{g})}^{\prime}$ by $U_{i} \cap Y_{(\mathrm{g})}^{\prime}=\left\{x_{1}=x_{2}=0, x_{i} \neq 0\right\}, 3 \leq i \leq 5 . \quad U_{i} \cap Y_{(\mathrm{g})}^{\prime}$ is the affine open set of $Y_{(\mathbf{g})}^{\prime}$ corresponding to the cone generated by the projection of $\left\{v_{j}, v_{k}: 3 \leq j, k \leq 5, j, k \neq i\right\}$. For instance $U_{5} \cap Y_{(\mathbf{g})}^{\prime}$ is generated by projection of $\left\{v_{3}, v_{4}\right\}$. The equations $\left\langle-m, v_{3}\right\rangle=5$ and $\left\langle-m, v_{4}\right\rangle=0$ have solution $-m=c_{1}+2 c_{2}$ in $M(\tau)$. Hence the divisor $5 D_{1} D_{2} D_{3}$ is given by the rational function $\chi^{\left(c_{1}+2 c_{2}\right)}$ on $U_{5} \cap Y_{(\mathbf{g})}$. Similarly, it is given by the rational functions $\chi^{c_{2}}$ on $U_{4} \cap Y_{(\mathbf{g})}^{\prime}$ and $\chi^{0}$ on $U_{3} \cap Y_{(\mathbf{g})}^{\prime}$. Hence the action of $\mathbf{T}$ on $L_{(\mathbf{g})}$ at the fixed points $q_{5}, q_{4}$ and $q_{3}$ has weights $\left(u_{1}+2 u_{2}\right), u_{2}$ and 0 respectively.

The orbifold line bundle $F_{(\mathbf{g})}$ is trivialized by the generator $\frac{\partial}{\partial\left(x_{2} / x_{i}\right)} \otimes\left(\zeta \bar{\omega}_{1}+\bar{\omega}_{2}\right)$ on $U_{i} \cap Y_{(\mathbf{g})}$. The transition maps $h_{i j}:\left(U_{j} \cap Y_{(\mathbf{g})}^{\prime}\right) \times \mathbb{C} \supset\left(U_{j} \cap U_{i} \cap Y_{(\mathbf{g})}^{\prime}\right) \times \mathbb{C} \rightarrow$ $\left(U_{j} \cap U_{i} \cap Y_{(\mathbf{g})}^{\prime}\right) \times \mathbb{C} \subset\left(U_{i} \cap Y_{(\mathbf{g})}^{\prime}\right) \times \mathbb{C}$ for $F_{(\mathbf{g})}^{\prime \prime}$ are given by $h_{j i}(x, c)=\left(x,\left(\frac{x_{j}}{x_{i}}\right)^{5}(x) c\right)$. Thus we can define a $\mathbf{T}$ action on $F_{(\mathrm{g})}^{\prime \prime}$ by:

(i) $t(x, c)=(t x, c)=\left(t x, \chi^{0}(t) c\right)$ for $t \in \mathbf{T}$ and $(x, c) \in\left(U_{5} \cap Y_{(\mathbf{g})}^{\prime}\right) \times \mathbb{C}$.

(ii) $t(x, c)=\left(t x,\left(\frac{x_{5}}{x_{4}}\right)^{5}(t) c\right)=\left(t x, \chi^{-c_{1}-c_{2}}(t) c\right)$ for $t \in \mathbf{T}$ and $(x, c) \in\left(U_{4} \cap\right.$ $\left.Y_{(\mathbf{g})}^{\prime}\right) \times \mathbb{C}$

(iii) $t(x, c)=\left(t x,\left(\frac{x_{5}}{x_{3}}\right)^{5}(t) c\right)=\left(t x, \chi^{-c_{1}-2 c_{2}}(t) c\right)$ for $t \in \mathbf{T}$ and $(x, c) \in\left(U_{3} \cap\right.$ $\left.Y_{(\mathbf{g})}^{\prime}\right) \times \mathbb{C}$

This action of $\mathbf{T}$ on $F_{(\mathbf{g})}^{\prime \prime}$ at the fixed points $q_{5}, q_{4}$ and $q_{3}$ has weights $0,\left(-u_{1}-\right.$ $\left.u_{2}\right)$ and $\left(-u_{1}-2 u_{2}\right)$ respectively.

Applying corollary 9.1.4 of [CK], which shows that for reduced orbifolds the localization formula has to be modified by dividing the contribution of each fixed point by the order of its local group, we have

$$
\begin{aligned}
& \int_{Y_{(\mathbf{g})}^{\prime}} e\left(F_{(\mathbf{g})}^{\prime \prime} \oplus L_{(\mathbf{g})}\right) \\
& =\frac{1}{25}\left(\frac{0\left(u_{1}+2 u_{2}\right)}{\left(\frac{1}{5} u_{1}+\frac{2}{5} u_{2}\right)\left(\frac{1}{5} u_{1}+\frac{1}{5} u_{2}\right)}+\frac{\left(-u_{1}-u_{2}\right) u_{2}}{\left(\frac{1}{5} u_{2}\right)\left(-\frac{1}{5} u_{1}-\frac{1}{5} u_{2}\right)}+\frac{\left(-u_{1}-2 u_{2}\right) 0}{\left(-\frac{1}{5} u_{2}\right)\left(-\frac{1}{5} u_{1}-\frac{2}{5} u_{2}\right)}\right) \\
& =1 .
\end{aligned}
$$

Finally by (5.8) and (5.9) we must have

$$
\int_{X_{(\mathbf{g})}}^{o r b} e\left(E_{(\mathbf{g})}\right)=\frac{1}{25}
$$

\section{References}

[AB] M.F. Atiyah and R. Bott: The moment map and equivariant cohomology, Topology 23 (1984), 1-28.

[Bai] W. Baily, Jr.: The decomposition theorem for $V$-manifolds, Amer. J. Math. 78 (1956), 862-888. 
[Bat] V. Batyrev: Dual polyhedra and mirror symmetry for Calabi-Yau hvpersurfaces in toric varieties, J. Algebraic Geom. 3 (1994), 493-535; also available at math.AG/9310003.

[BC] V. Batyrev and D. Cox: On the Hodge structure of projective hypersurfaces in toric varieties, Duke Math. J. 75 (1994), 293-338; also available at math.AG/9306011.

[BM] L. Borisov and A. Mavlyutov: String cohomology of Calabi-Yau hypersurfaces via Mirror Symmetry, math.AG/0109096.

[CG] J. Carlson and P. Griffiths: Infinitesimal variations of Hodge structure and the global Torelli problem, Jorneés de géométrie algébraic d'Angers, 1979, Sijthoff and Nordhoff, Alphen aan den Rijn, 1980, pp. 51-76.

[CR1] W. Chen and Y. Ruan: A new cohomology theory for orbifold, math.AG/0004129 v3.

[CR2] W. Chen and Y. Ruan: Orbifold Gromov-Witten theory, math.AG/0103156.

[CK] D.A. Cox and S. Katz: Mirror Symmetry and Algebraic Geometry. Mathematical Surveys and Monographs, 68. Amer. Math. Soc., Providence, RI, 1999.

[CLO] D. Cox, J. Little and D. O'Shea: Ideals, varieties, and algorithms. An introduction to computational algebraic geometry and commutative algebra. Second edition. SpringerVerlag, New York, 1997.

[Fu W. Fulton:Introduction to Toric Varieties. Annals of Mathematics Studies, 131. The William H. Roever Lectures in Geometry. Princeton Univ. Press, Princeton, NJ, 1993.

[GM] M. Goresky and R. MacPherson: Intersection homology theory, Topology 19 (1980), no. $2,135-162$.

[GP] B. Greene and M. Plesser: Duality in Calabi-Yau moduli space, Nuclear Physics B338 (1990), 15-37.

[Gr] P.A. Griffiths: Introduction to Algebraic Curves. Translated from the Chinese by Kuniko Weltin. Translations of Mathematical Monographs, 76. Amer. Math. Soc., Providence, RI, 1989.

[Ka] T. Kawasaki: The signature theorem for $V$-manifolds, Topology 17 (1978), 75-83.

[KN] S. Kobayashi and K. Nomizu: Foundations of Differential Geometry, Vol I. Interscience Publishers, a division of John Wiley \& Sons, New York, 1963.

[Ma] A.R. Mavlyutov: Semiample hypersurfaces in toric varieties, Duke Math. J. 101 (2000), no. 1, 85-116; also available at math.AG/9812163 v2.

[Od] T. Oda: Convex Bodies and Algebraic Geometry. Springer-Verlag, Berlin-Heidelberg-New York, 1988.

[Po] M. Poddar: Orbifold Hodge numbers of Calabi-Yau hypersurfaces, Pacific J. Math. (to appear), available at math.AG/0107152

[Ru] Y. Ruan: Cohomology ring of crepant resolutions of orbifolds, math.AG/0108195.

[Sc] P. Scott: The geometries of 3-manifolds, Bull. London Math. Soc. 15 (1983), no. 5, 401-487.

[Wo] S. Wolfram: The Mathematica ${ }^{\circledR}$ Book. Fourth edition. Wolfram Media, Inc., Champaign, IL; Cambridge University Press, Cambridge, 1999.

Department of Pure Mathematics, University of Waterloo, Waterloo, Ontario N2L 3G1, CANADA

E-mail address: bdpark@math.uwaterloo.ca

Department of Mathematics, Michigan State University, E. Lansing, Mi 48824, USA

E-mail address: poddar@math.msu.edu 